\title{
WIDE-FIELD INFRARED SURVEY EXPLORER OBSERVATIONS OF YOUNG STELLAR OBJECTS IN THE LYNDS 1509 DARK CLOUD IN AURIGA
}

\author{
Wilson M. Liu ${ }^{1,6}$, Deborah L. Padgett ${ }^{2}$, Susan Terebey ${ }^{3}$, John Angione ${ }^{3}$, Luisa M. Rebull $^{4}$, \\ Bruce McCollum ${ }^{1}$, Sergio Fajardo-Acosta ${ }^{1}$, and David Leisawitz ${ }^{5}$ \\ ${ }^{1}$ Infrared Processing and Analysis Center, California Institute of Technology, MC 100-22, Pasadena, CA 91125, USA; wliu@ipac.caltech.edu \\ ${ }^{2}$ National Aeronautics and Space Administration, Goddard Space Flight Center, Code 665, Greenbelt, MD 20771, USA \\ ${ }^{3}$ Department of Physics and Astronomy, California State University, Los Angeles, CA 90032, USA \\ ${ }^{4}$ Spitzer Science Center, California Institute of Technology, MC 314-6, Pasadena, CA 91125, USA \\ 5 National Aeronautics and Space Administration, Goddard Space Flight Center, Code 605, Greenbelt, MD 20771, USA \\ Received 2013 January 3; accepted 2014 March 14; published 2014 May 1
}

\begin{abstract}
The Wide-Field Infrared Survey Explorer (WISE) has uncovered a striking cluster of young stellar object (YSO) candidates associated with the L1509 dark cloud in Auriga. The WISE observations, at $3.4 \mu \mathrm{m}, 4.6 \mu \mathrm{m}, 12 \mu \mathrm{m}$, and $22 \mu \mathrm{m}$, show a number of objects with colors consistent with YSOs, and their spectral energy distributions suggest the presence of circumstellar dust emission, including numerous Class I, flat spectrum, and Class II objects. In general, the YSOs in L1509 are much more tightly clustered than YSOs in other dark clouds in the Taurus-Auriga star forming region, with Class I and flat spectrum objects confined to the densest aggregates, and Class II objects more sparsely distributed. We estimate a most probable distance of $485-700 \mathrm{pc}$, and possibly as far as the previously estimated distance of $2 \mathrm{kpc}$.
\end{abstract}

Key words: infrared: stars - stars: formation - stars: pre-main sequence

\section{INTRODUCTION}

Dark clouds (or dark nebulae) are distinct regions of high optical extinction known to contain dense, cold molecular gas and, in many cases, harboring active star formation. Well known studies cataloging these clouds were undertaken using optical observations (e.g., Barnard 1919; Lynds 1962). More recent decades have seen the use of infrared observations to probe the inner structure of these clouds and the protostars that lie within them (e.g., Lada et al. 2007; White et al. 2007).

The Lynds 1509 dark cloud is located in Auriga (R.A. = $05^{\mathrm{h}} 34^{\mathrm{m}} 12^{\mathrm{s}}$, decl. $=37^{\mathrm{d}} 17^{\mathrm{m}} 00^{\mathrm{s}}, \mathrm{J} 2000$ ), although it lies more than $10^{\circ}$ away from the well-studied Taurus-Auriga molecular cloud complex. The region is not contained within any existing Spitzer Space Telescope observations, including the GLIMPSE (Churchwell et al. 2009) and MIPSGAL (Carey et al. 2009) Galactic plane maps. L1509 is not well-studied, though the region (the surrounding $\sim 1 \mathrm{deg}^{2}$ ) appears in large-scale surveys for dust and molecular gas in the Milky Way, and contains a number of IRAS sources (Kawamura et al. 1998; Kiss et al. 2004). The region also contains several dark clouds and clumps identified by Dobashi et al. (2005). At optical wavelengths, L1509 covers approximately $0.1 \mathrm{deg}^{2}$ and has major and minor axes of about 20' (Lynds 1962; Dutra \& Bica 2002). The distance to L1509 is not well determined. A survey of ${ }^{13} \mathrm{CO}$ clouds in Auriga by Kawamura et al. (1998) cites a distance of $2 \mathrm{kpc}$ for molecular clouds in this region of Auriga, and in particular for their "Cloud 10" which they associate with L1509. We will further address the issue of distance in the current study.

Observations by the Wide-field Infrared Survey Explorer (WISE) have shown the L1509 cloud to contain a dramatic aggregate of bright 12 and $22 \mu \mathrm{m}$ sources. WISE conducted

\footnotetext{
${ }^{6}$ Current Address: WIYN Observatory, 950 North Cherry Avenue, Tucson,
} AZ 85719, USA. an all-sky survey in the mid-infrared (3.4-22 $\mu \mathrm{m}$; Wright et al. 2010; Cutri et al. 2012). The nature of the WISE data set makes it useful for probing regions of high visual extinction. In particular, Bands 3 and 4 (centered on 12 and $22 \mu \mathrm{m}$, respectively), which are sensitive to thermal emission from warm circumstellar dust, are ideal for investigating protostars embedded in dense material. The WISE data, when combined with the Two Micron All Sky Survey (2MASS; Skrutskie et al. 2006), allows one to characterize the spectral energy distribution (SED) of an object in seven bands from 1.2 to $22 \mu \mathrm{m}$. Data from the WISE survey have already been used to investigate several star forming regions (e.g., Koenig et al. 2012; Liu et al. 2011; Rebull et al. 2011b).

In Section 2 we will describe the WISE observations of L1509, as well as new near-infrared spectroscopic follow-up observations of young stellar object (YSO) candidates. We will present results of our photometric and spectroscopic analyses in Section 3 and discuss them in the context of other star forming regions in Section 4. Our key conclusions are presented in Section 5.

\section{OBSERVATIONS AND DATA REDUCTION}

\subsection{WISE}

WISE completed its cryogenic mission between 2010 January and 2010 August, during which time the entire sky was surveyed in four wavelength bands. Observations of L1509 and the surrounding region took place 2010 March 9 and 10. Single WISE frames are coadded to create the Atlas images. The typical depth-of-coverage for the L1509 region is 13 single frames, resulting in total exposure times of approximately $100 \mathrm{~s}$ in Bands 1 ( $3.4 \mu \mathrm{m}$; hereafter $W 1)$ and $2(4.6 \mu \mathrm{m}, W 2)$ and $114 \mathrm{~s} \mathrm{in}$ Bands $3(12 \mu \mathrm{m}, W 3)$ and $4(22 \mu \mathrm{m}, W 4)$. The $5 \sigma$ point source sensitivities for the survey, at the ecliptic, are $0.08,0.11,1$, and $6 \mathrm{mJy}$ in $W 1-W 4$, respectively, though the sensitivity of the L1509 observations should be deeper, as it lies off the 

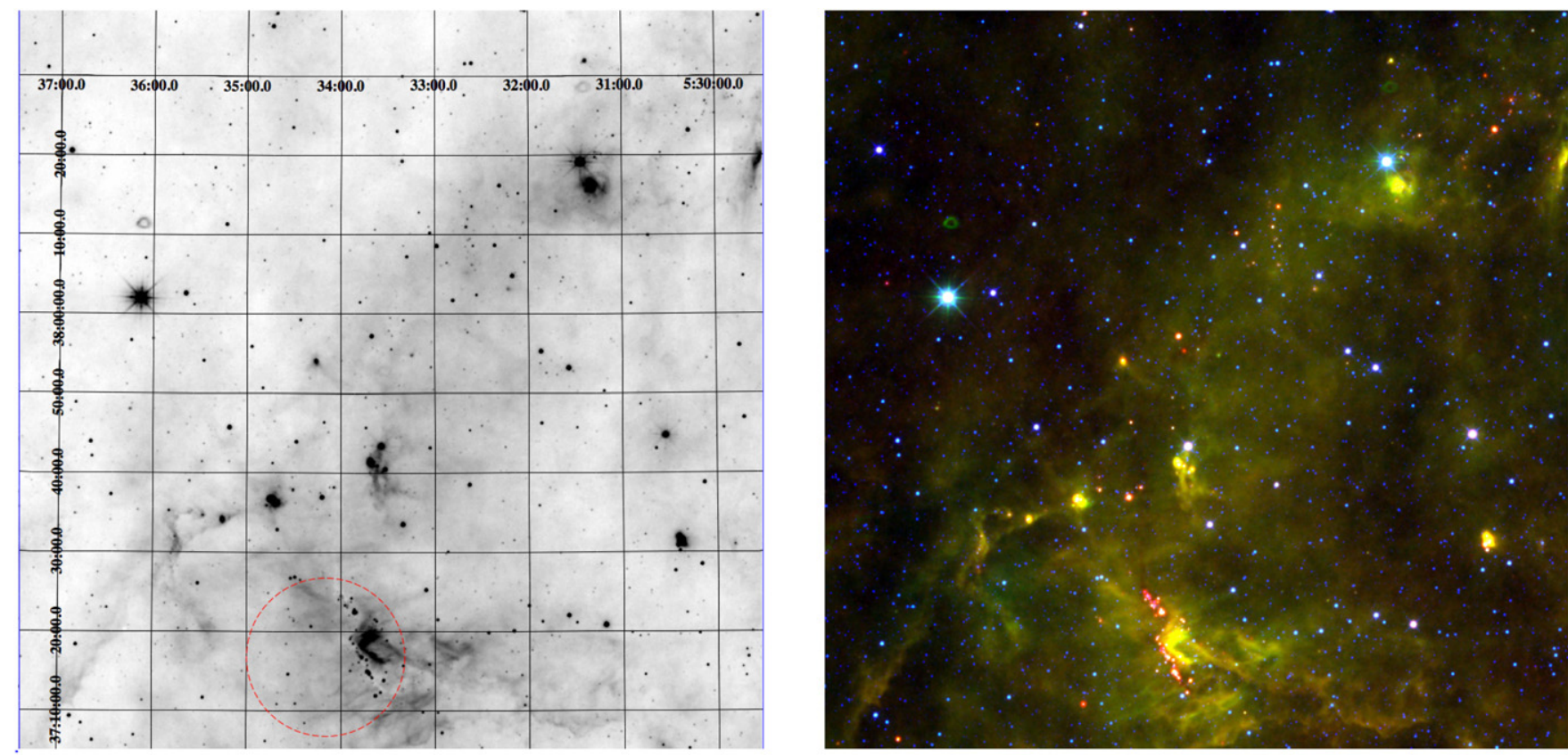

Figure 1. Left: Band $3(12 \mu \mathrm{m})$ image of the WISE Atlas tile containing the L1509 cluster. The cluster is located at the bottom-center of the image. The dotted red circle indicates the approximate extent of the optical dark cloud. Right: three-color image of the same atlas tile, with $W 2, W 3$, and $W 4$ mapped into blue, green, and red, respectively. North is up and east is to the left.

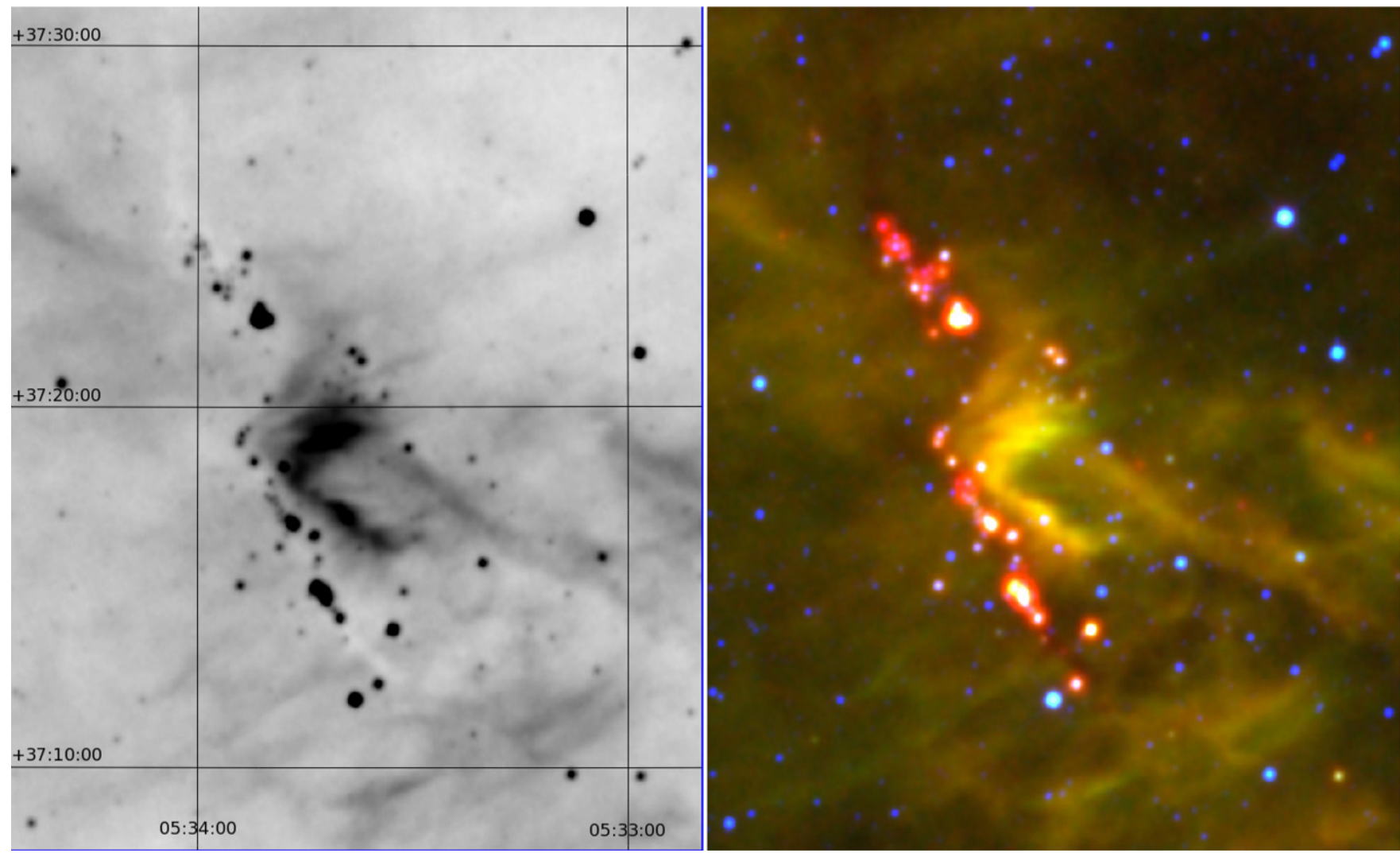

Figure 2. Left; zoomed $W 3(12 \mu \mathrm{m})$ image of the L1509 cluster. Right: three-color image of the cluster, with $W 2, W 3$, and $W 4$ mapped into blue, green, and red, respectively.

ecliptic plane. Angular resolution for WISE images is $\sim 6^{\prime \prime}$ in $W 1, W 2$, and $W 3$, and $12^{\prime \prime}$ in W4. Figure 1 shows the WISE atlas image containing the L1509 dark cloud and the associated cluster of bright $12 \mu \mathrm{m}$ and $22 \mu \mathrm{m}$ sources. The atlas tile is about 1.56 on a side. A zoomed image of the cluster is shown in Figure 2.

\subsection{Palomar Hale 200 inch with Triple Spectrograph}

We have also obtained near-infrared spectra for 19 of the YSO candidates in the cluster, using the Triple Spectrograph instrument (hereafter Triplespec) at the Hale telescope on Palomar Mountain. Triplespec provides simultaneous $J$-, $H$-, 


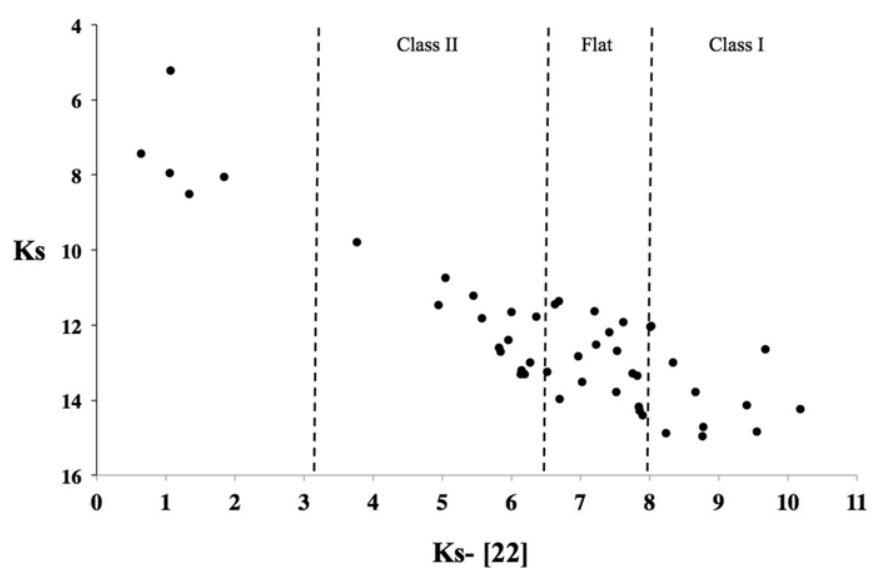

Figure 3. $K_{S}$ vs. $K_{S}-[22]$ color-magnitude diagram for 45 candidate YSOs that have photometry in both bands. We denote the color regions for each SED class.

and $K$-band spectroscopy with a spectral resolution of $R \sim$ 2500. The slit size of Triplespec is $1^{\prime \prime} \times 30^{\prime \prime}$. Objects blended in the WISE image were generally fairly well separated with the spatial resolution provided by Hale/Triplespec. Full results from these data will be presented in a future paper. In this paper, we present example spectra, note objects with strong emission lines, and we refer to the spectra in order to make an estimate for the distance to the cluster in Section 4.

Reduction and calibration of spectra were completed using IRAF, in a manner identical to Angione (2012). To summarize: background features, including sky lines and thermal background, were subtracted from the images using sky frames.
Telluric features were removed using early A0V type standard star spectra, which allow us to isolate atmospheric lines and divide them out of the raw spectrum. Raw data from Triplespec feature a curve in the spectra, in a direction orthogonal to the dispersion direction. The spectra were rectified using nonlinear fits in the $x$ and $y$ directions on the detector. Wavelength calibration was performed using $\mathrm{OH}$ sky lines, and was performed separately for data taken on different nights.

\section{RESULTS}

The WISE images show a well defined aggregate of bright 12 and $22 \mu \mathrm{m}$ objects, suggestive of YSOs, along a dark filament in the infrared-bright nebulosity at the bottom center of Figure 1. The densest cluster of YSOs stretches about 15 arcmin in a roughly northeast to southwest direction. At visible wavelengths, the cloud is located at R.A. = $05^{\mathrm{h}} 34^{\mathrm{m}} 12^{\mathrm{s}} \quad$ decl. $=37^{\mathrm{d}} 17^{\mathrm{m}} 00^{\mathrm{s}} \quad(\mathrm{J} 2000)$, about 15 arcmin east of the center of the dense cluster, and has a spatial extent of about $20 \times 20 \mathrm{arcmin}$. The WISE observations show the infrared-bright nebulosity continues to the north and west for the entire length of the WISE tile, about 1.5. A dark filament in the infrared can be traced for roughly half the extent of the image. There are also red objects well to the north of the dense aggregate, suggesting star formation in a more dispersed mode or migration well away from the L1509 cluster. For this study, we focus on the dense cluster of objects closest to the L1509 cloud (Figure 2).

We searched the WISE All-Sky Release catalog using the GATOR query tool provided by the NASA/IPAC Infrared

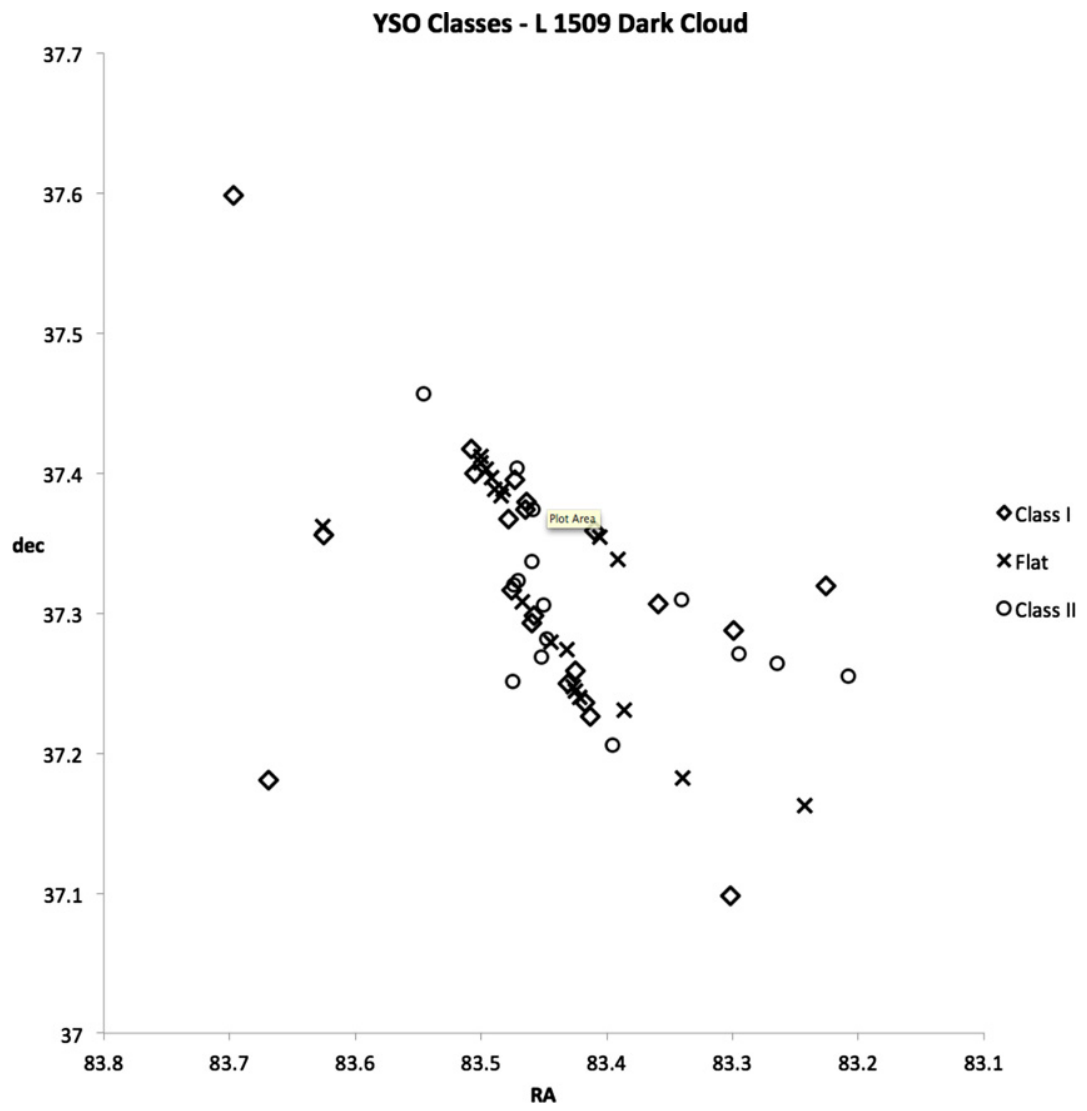

Figure 4. Map of the L1509 YSO cluster with the SED classes of each candidate denoted. 


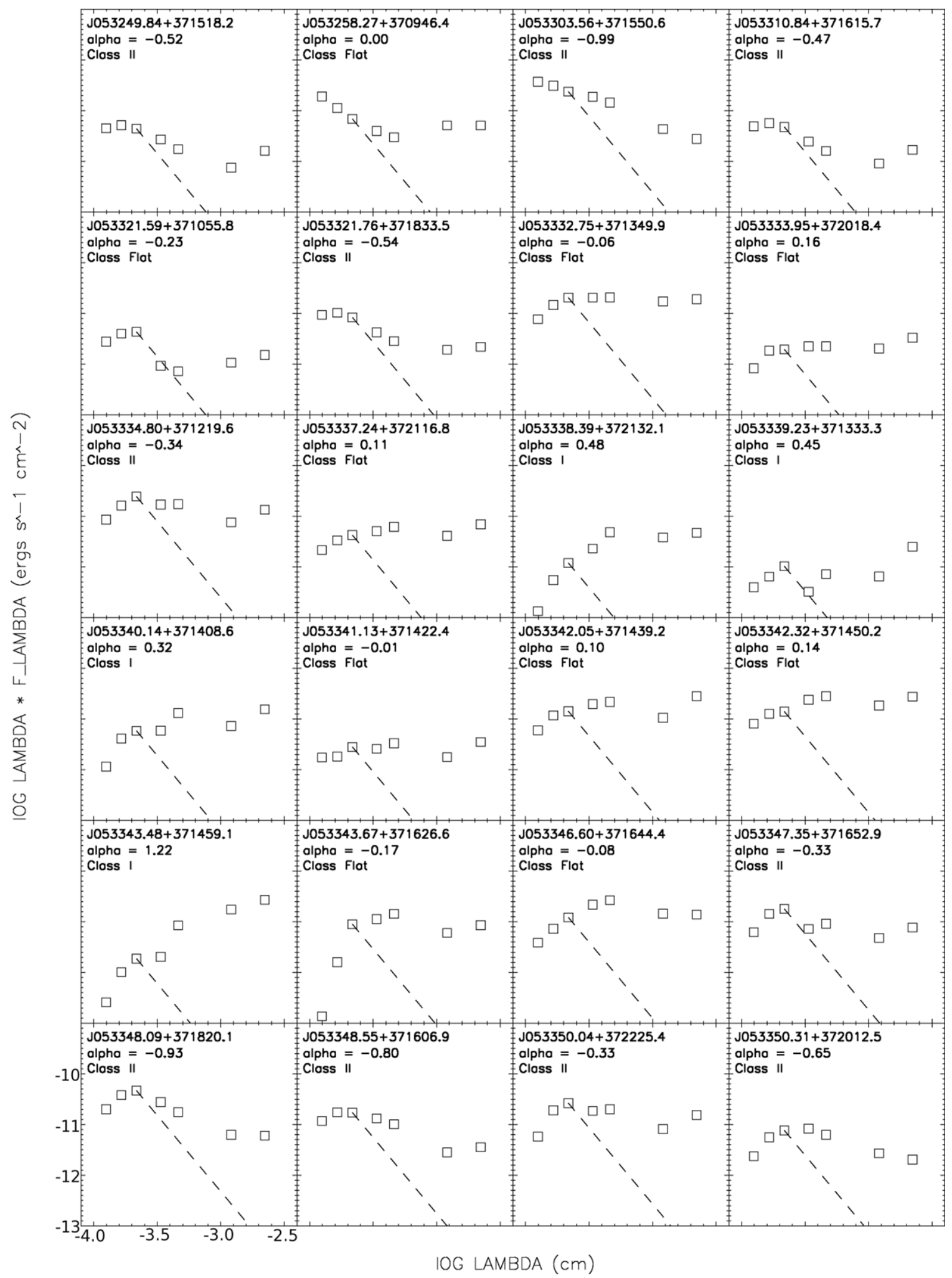

Figure 5. Spectral energy distributions of 41 Class I, Class II, and flat spectrum objects with seven band (WISE + 2MASS) photometric data. The dashed line indicates the Rayleigh-Jeans expectation for photospheric emission, normalized to the 2MASS $K_{S}$-band point. The photometric errors are typically less than $10 \%$ with the exception of the crowded and nebulosity-embedded objects described above. The SEDs are ordered by R.A.

Science Archive. ${ }^{7}$ Our initial positional search selected objects with R.A. between $\alpha=05^{\mathrm{h}} 32^{\mathrm{m}} 48^{\mathrm{s}}$ and $05^{\mathrm{h}} 35^{\mathrm{m}} 12^{\mathrm{s}}$ and decl. between $\delta=+37^{\mathrm{d}} 04^{\mathrm{m}} 48^{\mathrm{s}}$ and $+37^{\mathrm{d}} 36^{\mathrm{m}} 00^{\mathrm{s}}$. This search returned

\footnotetext{
7 http://irsa.ipac.caltech.edu/Missions/wise.html
}

6594 sources in the vicinity of the YSO cluster seen in Figure 2. Many of these objects are detections of non-point source emission originating from the extensive nebulosity in the region. In order to select relatively red, point-like objects, we chose objects that are well-detected in $W 4$, using a criteria of $\mathrm{S} / \mathrm{N}>10$. 


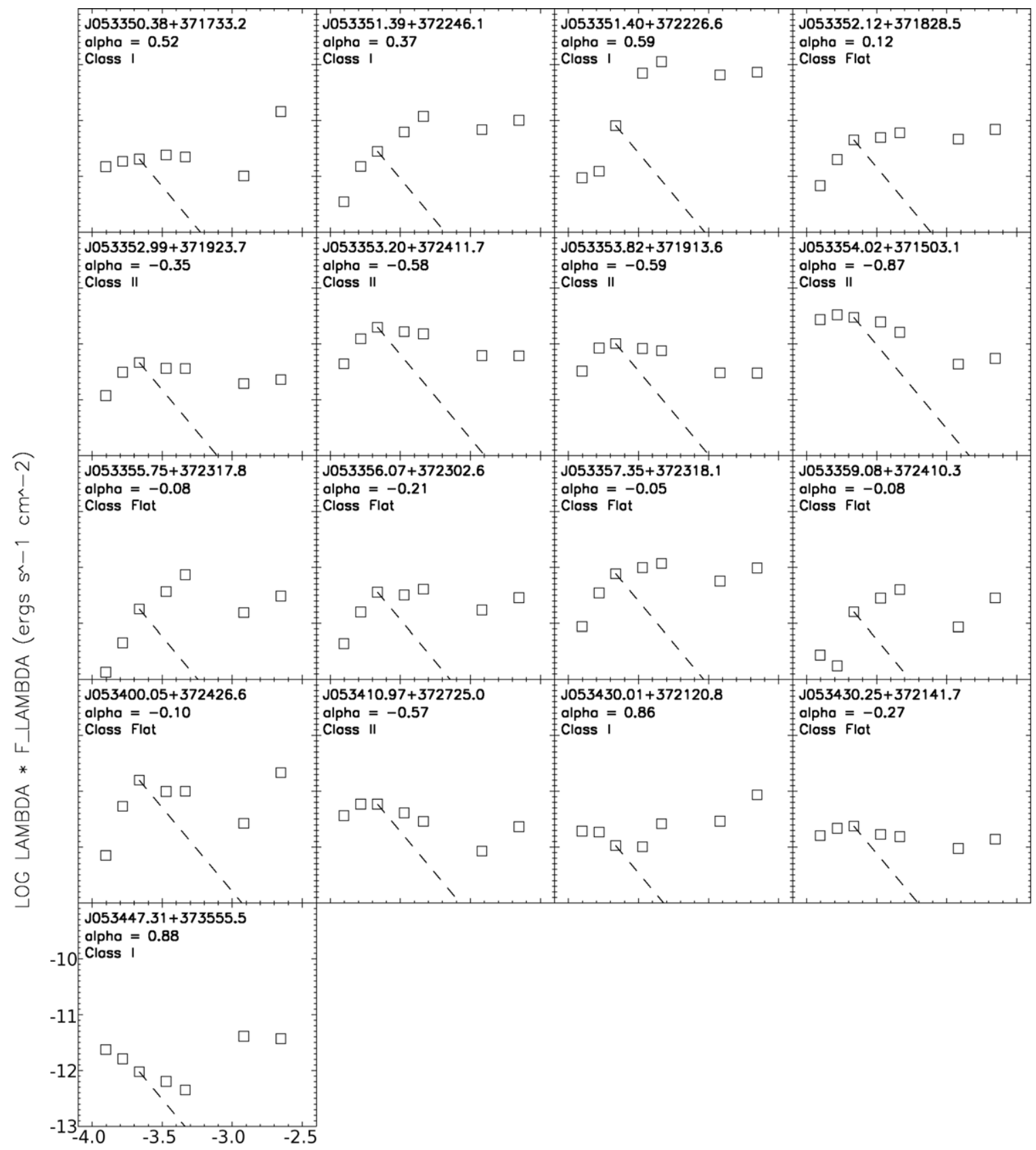

LOG LAMBDA (cm)

Figure 5. (Continued)

We required that all objects have a high-confidence color excess in $K-$ [22] or [3.4] - [22], with a value greater than $5 \sigma$ above the photosphere. Additionally, we selected objects that have a low photometric error, with an error in magnitude of less than 0.2 . These selection criteria did a reasonably good job of identifying the YSOs visible in the image (Figure 2), though contamination due to extractions on nebulosity was still a problem. These contaminants were removed by visual inspection of the image. The final result is a sample of 61 YSO candidates.

In Figure 3 we plot the $\left[K_{S}\right]-[22]$ color-magnitude diagram for 45 sources with photometry in both bands. Unreddened main sequence stars have zero color, with YSO candidates located to the right and down from the main sequence locus. The level of mid-infrared excess for each object can be quantified using its SED slope. Following Wilking et al. (2001), objects are placed into YSO classes using the value of $\alpha_{\mathrm{IR}}$ which is defined as $d \log \lambda F_{\lambda} / d \log \lambda$. Class I objects, the reddest, have $\alpha_{\mathrm{IR}}>0.3$. Flat spectrum sources have values near zero $\left(-0.3<\alpha_{\mathrm{IR}}<0.3\right)$ and Class II sources have $\alpha_{\mathrm{IR}}<-0.3$. For each of the objects, we use all points between $K$-band and $22 \mu \mathrm{m}$, inclusive, and perform a simple linear fit to calculate $\alpha_{\mathrm{IR}}$. For 14 objects, there was no $K$-band detection; in those cases we use the four WISE bands to calculate $\alpha_{\mathrm{IR}}$. In Figure 4 we plot the locations of the detected YSOs on a map, noting the class of the objects based upon the derived values of $\alpha_{\mathrm{IR}}$. In total, there are 21 Class I objects, 19 flat spectrum objects, and 15 Class II objects. 


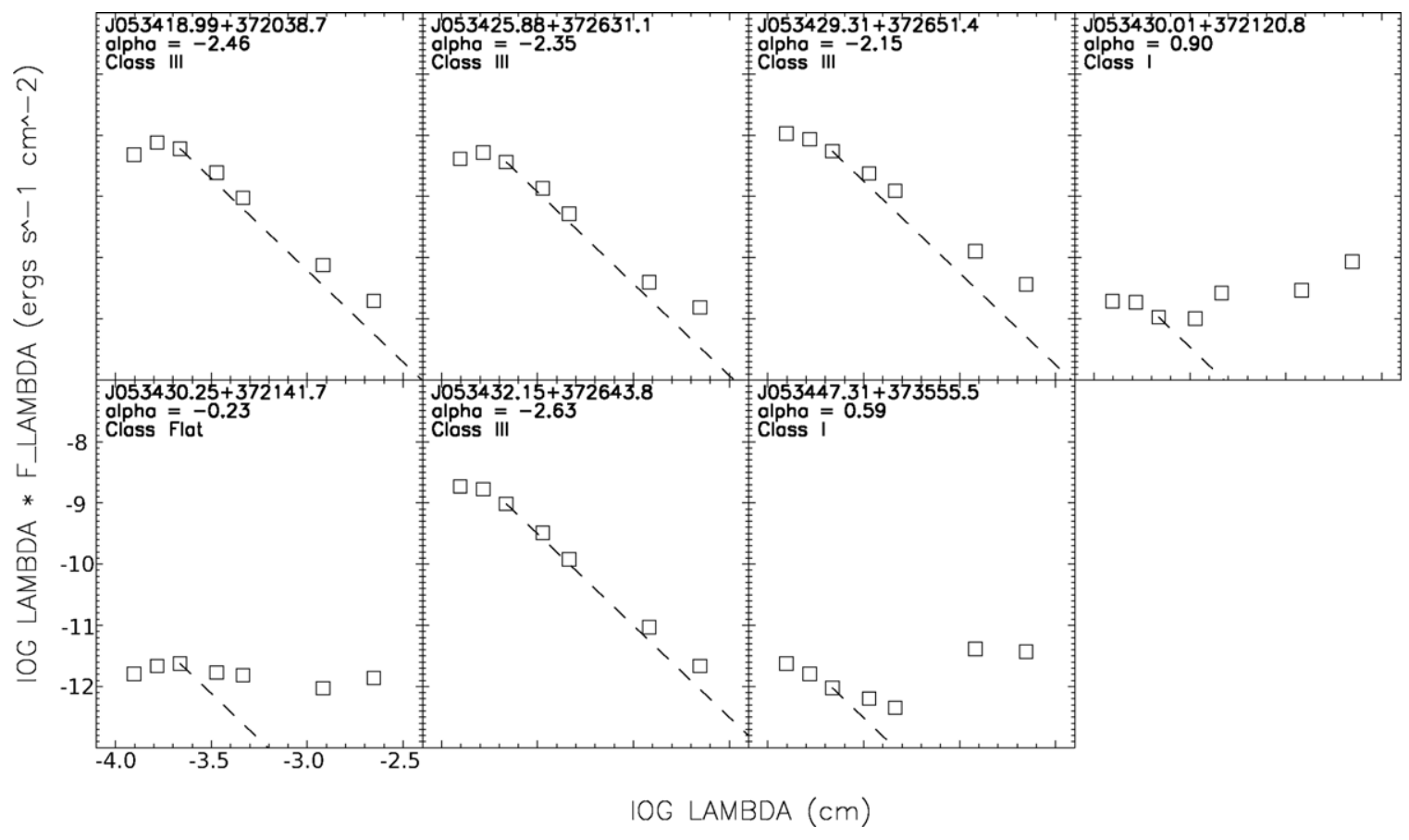

Figure 5. (Continued)

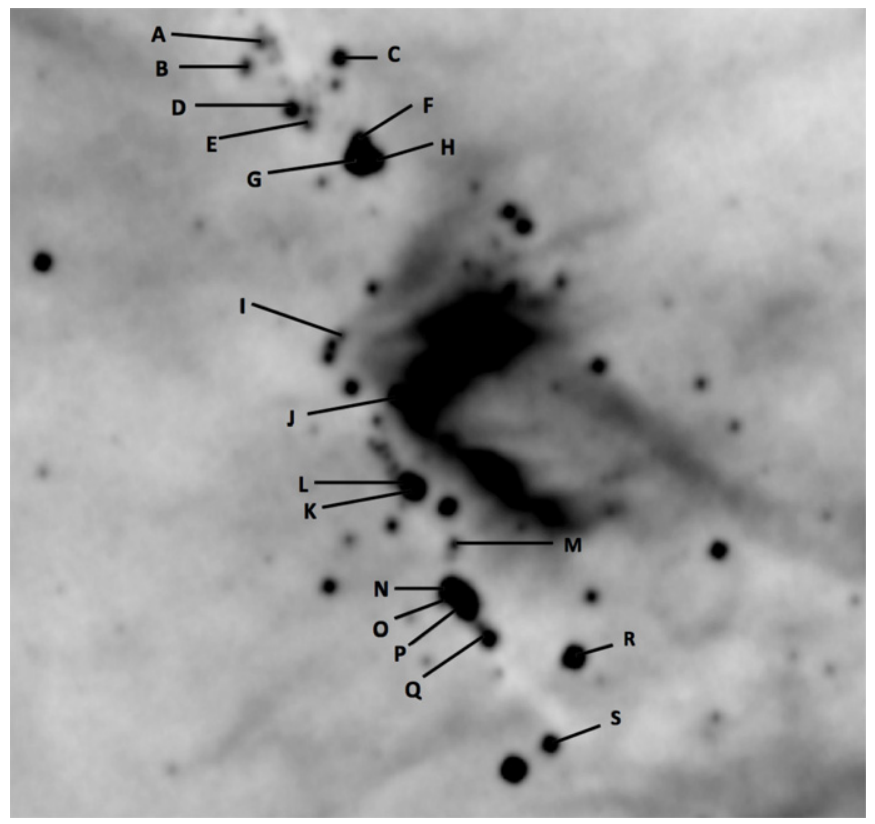

Figure 6. Map showing Band $3(12 \mu \mathrm{m})$ image of the cluster, with the spectroscopy targets designated "A"-"S".

Several objects are designated as Class III (not shown on the map) and have much smaller levels of excess (see Table 1). It is quite possible that one or more of these are actually reddened background stars not physically associated with the cluster.

For the 61 YSO candidates in the final sample, we derive flux densities at each wavelength based upon the WISE photometry. These values can be found in Table 1. Each of the YSO candidates was examined visually in each of the four WISE bands to note which of the objects were subject to possible photometric contamination from crowding or bright nebulosity. These objects are indicated in the table under the "Notes" field. For crowded objects (i.e., objects with another extracted point source within its point-spread function, denoted "C") the majority are subject to "active deblending" in the WISE photometry pipeline (see Cutri et al. 2012 for a detailed description of the WISE data pipeline). We also note objects which are deeply embedded in nebulosity (denoted "N"), where the background is of significant brightness compared to the object itself. Objects with an "N" or "C" flag may be subject to photometric errors in addition to those quoted in the flux fields, by perhaps as much as a few tens of percent in extreme cases. For 40 Class I, Class II, and flat spectrum objects with seven bands of photometry (2MASS + WISE), we plot their SEDs in Figure 5. In general, the photometric errors are less than $10 \%$, with the exception of the crowded and nebulosity-embedded objects described above. In Table 1 we also note objects that display emission lines in their spectra. Objects with a " $\mathrm{Pa} \beta$," "Br," or " $\mathrm{H}_{2}$ " have emission lines at $\mathrm{Pa} \beta(1.282 \mu \mathrm{m})$, in the Brackett series, and $\mathrm{H}_{2}(2.122 \mu \mathrm{m})$, respectively.

Spectra were obtained for 19 objects in the cluster. A map denoting the 19 objects is shown in Figure 6. The objects are also indicated in Table 1 under the "Notes" column. Figures 7-24 present the spectra for the objects. Although the Triplespec instrument takes simultaneous spectra in $J$-, $H$-, and $K$-bands, we include only the bands for which $\mathrm{S} / \mathrm{N}>20$. Sufficient signal-to-noise ratio $(\mathrm{S} / \mathrm{N})$ was not achieved for J053343.48+371459.1 (Object "N"), so it is not included. Five objects (J053400.05+372426.6, J053401.29+ 372357.9, J053356.07+372302.6, J053350.04+372225.4, and J053340.14+371408.6) in the cluster display pronounced CO absorption (from 2.3 to $2.4 \mu \mathrm{m})$ as well as $\mathrm{Na} \mathrm{I}(2.21 \mu \mathrm{m})$ and Ca I $(2.26 \mu \mathrm{m})$ absorption, with equivalent widths consistent with the dwarf locus (Greene \& Lada 1996). Following Greene \& Lada, these lines can provide a rough estimate of spectral type for these YSOs. These five objects appear to have absorption strengths consistent with early- to mid-K-type (K0-K7) stars. Table 2 lists these stars and the equivalent widths of the lines used to estimate their spectral types. 
Table 1

YSO Candidates in the L1509 Cloud

\begin{tabular}{|c|c|c|c|c|c|c|c|c|c|c|}
\hline$\overline{\text { Source ID }}{ }^{\mathrm{a}}$ & $\begin{array}{c}F_{J}^{\mathrm{b}} \\
(\mathrm{mJy})\end{array}$ & $\overline{F_{H}}$ & $F_{K s}$ & $F_{3.4}$ & $F_{4.6}$ & $F_{12}$ & $F_{22}$ & $\alpha_{\mathrm{IR}}$ & Class & Notes $^{\mathrm{c}}$ \\
\hline J053249.84+371518.2 & $1.8 \pm 0.065$ & $2.9 \pm 0.095$ & $3.2 \pm 0.11$ & $3.1 \pm 0.07$ & $2.7 \pm 0.059$ & $3 \pm 0.2$ & $12 \pm 1.1$ & -0.52 & II & $\mathrm{N}$ \\
\hline J053254.15+371909.0 & $\ldots$ & $\cdots$ & $\cdots$ & $0.13 \pm 0.012$ & $0.29 \pm 0.02$ & $0.51 \mathrm{u}$ & $19 \pm 1$ & 1.30 & I & $\mathrm{N}, \mathrm{C}$ \\
\hline J053258.27+370946.4 & $7.8 \mathrm{u}$ & $6.3 \pm 0.16$ & $5 \pm 0.37$ & $4.5 \pm 0.1$ & $4.6 \pm 0.093$ & $21 \pm 0.4$ & $38 \pm 1.4$ & 0.00 & Flat & \\
\hline J053303.56+371550.6 & $15 \pm 0.3$ & $17 \pm 0.25$ & $17 \pm 0.29$ & $21 \pm 0.45$ & $22 \pm 0.39$ & $18 \pm 0.45$ & $20 \pm 1.2$ & -0.99 & II & $\mathrm{N}$ \\
\hline J053310.84+371615.7 & $2 \pm 0.069$ & $3.2 \pm 0.096$ & $3.5 \pm 0.12$ & $2.8 \pm 0.061$ & $2.5 \pm 0.05$ & $3.7 \pm 0.21$ & $12 \pm 1.2$ & -0.47 & II & $\mathrm{N}$ \\
\hline J053311.86+371715.5 & $\cdots$ & $\cdots$ & $\cdots$ & $0.32 \pm 0.013$ & $0.97 \pm 0.029$ & $0.41 \mathrm{u}$ & $18 \pm 1.2$ & 0.58 & I & $\mathrm{N}$ \\
\hline J053312.48+370554.5 & $\cdots$ & $\cdots$ & $\cdots$ & $0.47 \pm 0.018$ & $1.3 \pm 0.034$ & $2.1 \pm 0.19$ & $31 \pm 1.4$ & 0.91 & I & \\
\hline J053320.47+373331.5 & $2200 \pm 37$ & $4700 \pm 78$ & $5400 \pm 79$ & $3100 \pm 190$ & $2500 \pm 99$ & $480 \pm 6.2$ & $180 \pm 4.1$ & -2.48 & III & \\
\hline J053321.59+371055.8 & $1.1 \pm 0.061$ & $2.2 \pm 0.088$ & $3.1 \pm 0.12$ & $1 \pm 0.029$ & $1.1 \pm 0.032$ & $4.3 \pm 0.2$ & $11 \pm 1.1$ & -0.23 & Flat & $\mathrm{N}$ \\
\hline J053321.76+371833.5 & $3.9 \pm 0.092$ & $5.7 \pm 0.11$ & $6 \pm 0.14$ & $4.8 \pm 0.11$ & $4.4 \pm 0.093$ & $7.7 \pm 0.27$ & $16 \pm 1.1$ & -0.54 & II & $\mathrm{N}$ \\
\hline J053326.08+371822.6 & $\cdots$ & $\cdots$ & $\cdots$ & $0.053 \pm 0.0085$ & $0.25 \pm 0.017$ & $0.6 \mathrm{u}$ & $16 \pm 1.1$ & 1.62 & I & $\mathrm{N}$ \\
\hline J053332.75+371349.9 & $3.2 \pm 0.087$ & $8.2 \pm 0.15$ & $15 \pm 0.25$ & $23 \pm 0.51$ & $32 \pm 0.53$ & $70 \pm 0.96$ & $140 \pm 2.9$ & -0.06 & Flat & $\mathrm{Br}, \mathrm{Pa} \beta$, Sp.Obj. “R” \\
\hline J053333.95+372018.4 & $0.34 \mathrm{u}$ & $1 \pm 0.079$ & $1.4 \pm 0.099$ & $2.5 \pm 0.063$ & $3.4 \pm 0.076$ & $8.2 \pm 0.29$ & $25 \pm 1.6$ & 0.16 & Flat & $\mathrm{N}, \mathrm{C}$ \\
\hline J053334.80+371219.6 & $3.5 \pm 0.091$ & $9 \pm 0.15$ & $18 \pm 0.28$ & $19 \pm 0.42$ & $27 \pm 0.47$ & $31 \pm 0.53$ & $99 \pm 2.5$ & -0.34 & II & $\mathrm{H}_{2}, \mathrm{Br}, \mathrm{Pa} \beta, \mathrm{CO}$ emiss., Sp.Obj. "S" \\
\hline J053337.24+372116.8 & $0.89 \pm 0.056$ & $1.9 \pm 0.09$ & $3.1 \pm 0.11$ & $5.7 \pm 0.14$ & $9.5 \pm 0.2$ & $17 \pm 0.44$ & $51 \pm 2.4$ & 0.11 & Flat & $\mathrm{C}$ \\
\hline J053338.39+372132.1 & $0.055 \mathrm{u}$ & $0.31 \mathrm{u}$ & $0.87 \pm 0.092$ & $2.6 \pm 0.06$ & $7.5 \pm 0.15$ & $15 \pm 0.44$ & $35 \pm 2.1$ & 0.48 & I & \\
\hline J053339.23+371333.3 & $0.16 \mathrm{u}$ & $0.36 \mathrm{u}$ & $0.75 \pm 0.1$ & $0.36 \pm 0.019$ & $1.1 \pm 0.04$ & $2.6 \pm 0.2$ & $18 \pm 1.7$ & 0.45 & I & $\mathrm{C}$ \\
\hline J053339.26+371651.9 & $32 \pm 0.53$ & $75 \pm 1$ & $81 \pm 1.1$ & $50 \pm 1.1$ & $28 \pm 0.55$ & $16 \pm 0.42$ & $32 \pm 2$ & -1.45 & III & $\mathrm{N}$ \\
\hline J053340.14+371408.6 & $0.47 \pm 0.052$ & $2.3 \pm 0.094$ & $4.2 \pm 0.13$ & $6.6 \pm 0.15$ & $20 \pm 0.35$ & $29 \pm 0.48$ & $110 \pm 2.5$ & 0.32 & I & C, Sp.Obj. “Q” \\
\hline J053341.13+371422.4 & $0.72 \mathrm{u}$ & $1 \pm 0.089$ & $2 u$ & $2.9 \pm 0.07$ & $5.1 \pm 0.1$ & $7.1 \pm 0.24$ & $26 \pm 1.3$ & -0.01 & Flat & $\mathrm{C}$ \\
\hline J053342.01+371532.5 & $\ldots$ & $\ldots$ & $\ldots$ & $0.15 \pm 0.01$ & $0.73 \pm 0.028$ & $0.37 \mathrm{u}$ & $21 \pm 1.5$ & 1.00 & I & $\mathrm{C}$ \\
\hline J053342.05+371439.2 & $2.5 \pm 0.095$ & $6.5 \pm 0.2$ & $10 \pm 0.3$ & $22 \pm 0.47$ & $33 \pm 0.58$ & $43 \pm 0.67$ & $210 \pm 3.8$ & 0.10 & Flat & $\mathrm{C}, \mathrm{H}_{2}, \mathrm{~Pa} \beta$, Sp.Obj. "P" \\
\hline J053342.32+371450.2 & $3.3 \pm 0.1$ & $7 \pm 0.17$ & $10 \pm 0.24$ & $27 \pm 0.62$ & $43 \pm 0.84$ & $74 \pm 1.2$ & $200 \pm 5.8$ & 0.14 & Flat & $\mathrm{C}, \mathrm{H}_{2}$, Sp.Obj. "O" \\
\hline J053343.48+371459.1 & $0.11 \mathrm{u}$ & $0.56 \mathrm{u}$ & $1.4 \pm 0.1$ & $2.3 \pm 0.055$ & $13 \pm 0.23$ & $70 \pm 0.97$ & $200 \pm 3.9$ & 1.22 & I & $\mathrm{C}, \mathrm{H}_{2}$, Sp.Obj. "N" \\
\hline J053343.67+371626.6 & $0.056 \mathrm{u}$ & $0.89 \pm 0.081$ & $6.5 \pm 0.17$ & $13 \pm 0.28$ & $22 \pm 0.41$ & $24 \pm 0.54$ & $63 \pm 4.1$ & -0.17 & Flat & $\mathrm{C}$ \\
\hline J053346.60+371644.4 & $1.6 \mathrm{u}$ & $4 \pm 0.12$ & $8.8 \pm 0.24$ & $24 \pm 0.52$ & $41 \pm 0.8$ & $58 \pm 0.91$ & $100 \pm 3.7$ & -0.08 & Flat & C, Sp.Obj. "L" \\
\hline J053347.35+371652.9 & $2.6 \pm 0.1$ & $8 \mathrm{u}$ & $13 u$ & $8.1 \pm 0.17$ & $14 \pm 0.27$ & $19 \pm 0.41$ & $57 \pm 2.7$ & -0.33 & II & C, Sp.Obj. "K" \\
\hline J053348.09+371820.1 & $8.2 \pm 0.16$ & $21 \pm 0.31$ & $34 \pm 0.5$ & $31 \pm 0.69$ & $27 \pm 0.52$ & $25 \pm 0.72$ & $45 \pm 2.3$ & -0.93 & II & $\mathrm{N}, \mathrm{Pa} \beta$, Sp.Obj. "J" \\
\hline J053348.55+371606.9 & $4.9 \pm 0.13$ & $9.6 \pm 0.2$ & $12 \pm 0.27$ & $15 \pm 0.31$ & $16 \pm 0.29$ & $11 \pm 0.31$ & $26 \pm 1.4$ & -0.80 & II & \\
\hline J053349.91+371754.7 & $\cdots$ & $\cdots$ & $\cdots$ & $1.1 \pm 0.034$ & $6.6 \pm 0.13$ & $7.4 \pm 0.26$ & $69 \pm 2.9$ & 0.76 & I & $\mathrm{C}$ \\
\hline J053350.04+372225.4 & $2.4 \pm 0.077$ & $11 \pm 0.18$ & $19 \pm 0.34$ & $21 \pm 0.42$ & $31 \pm 0.6$ & $33 \pm 0.6$ & $110 \pm 2.6$ & -0.33 & II & $\mathrm{C}, \mathrm{H}_{2}$, Sp.Obj. "H" \\
\hline J053350.31+372012.5 & $0.98 \pm 0.058$ & $3.1 \pm 0.092$ & $5.5 \pm 0.14$ & $9.3 \pm 0.21$ & $9.7 \pm 0.2$ & $11 \pm 0.31$ & $15 \pm 1.5$ & -0.65 & II & \\
\hline J053350.38+371733.2 & $0.62 \pm 0.056$ & $1 \pm 0.083$ & $1.5 \pm 0.098$ & $2.7 \pm 0.095$ & $3.4 \pm 0.088$ & $4.1 \pm 0.23$ & $110 \pm 3.2$ & 0.52 & I & $\mathrm{C}$ \\
\hline J053351.39+372246.1 & $0.15 \mathrm{u}$ & $0.83 \mathrm{u}$ & $2 \pm 0.1$ & $7 \pm 0.16$ & $18 \pm 0.37$ & $28 \pm 0.56$ & $75 \pm 2.3$ & 0.37 & I & $\mathrm{C}, \mathrm{H}_{2}$, Sp.Obj. "F" \\
\hline J053351.40+372226.6 & $0.39 \mathrm{u}$ & $0.69 \pm 0.079$ & $5.9 \pm 0.14$ & $79 \pm 1.7$ & $170 \pm 3.4$ & $260 \pm 3.4$ & $540 \pm 8$ & 0.59 & I & $\mathrm{C}, \mathrm{H}_{2}$, Sp.Obj. "G" \\
\hline J053352.12+371828.5 & $0.28 \mathrm{u}$ & $1.1 \pm 0.08$ & $3.3 \pm 0.11$ & $5.6 \pm 0.13$ & $9.3 \pm 0.19$ & $19 \pm 0.48$ & $51 \pm 2.2$ & 0.12 & Flat & \\
\hline J053352.99+371923.7 & $0.49 \pm 0.055$ & $1.7 \pm 0.081$ & $3.4 \pm 0.12$ & $4.1 \pm 0.095$ & $5.6 \pm 0.11$ & $7.8 \pm 0.24$ & $17 \pm 1.4$ & -0.35 & II & $\mathrm{C}, \mathrm{H}_{2}$, Sp.Obj. "I" \\
\hline
\end{tabular}


Table 1

(Continued)

\begin{tabular}{|c|c|c|c|c|c|c|c|c|c|c|}
\hline Source ID ${ }^{\mathrm{a}}$ & $\begin{array}{c}F_{J}^{\mathrm{b}} \\
(\mathrm{mJy})\end{array}$ & $F_{H}$ & $F_{K s}$ & $F_{3.4}$ & $F_{4.6}$ & $F_{12}$ & $F_{22}$ & $\alpha_{\mathrm{IR}}$ & Class & Notes $^{\mathrm{c}}$ \\
\hline J053353.20+372411.7 & $1.8 \pm 0.067$ & $6.9 \pm 0.13$ & $14 \pm 0.24$ & $19 \pm 0.38$ & $23 \pm 0.45$ & $25 \pm 0.44$ & $45 \pm 1.5$ & -0.58 & II & Sp.Obj. "B" \\
\hline J053353.58+372343.4 & $\ldots$ & $\ldots$ & $\ldots$ & $0.16 \pm 0.022$ & $1.3 \pm 0.062$ & $7.1 \pm 0.36$ & $56 \pm 4.3$ & 1.78 & I & $\mathrm{C}$ \\
\hline J053353.82+371913.6 & $1.3 \pm 0.06$ & $4.7 \pm 0.11$ & $7.4 \pm 0.16$ & $9.3 \pm 0.22$ & $12 \pm 0.26$ & $12 \pm 0.28$ & $22 \pm 1.7$ & -0.59 & II & $\mathrm{C}$ \\
\hline J053354.02+371503.1 & $11 \pm 0.21$ & $18 \pm 0.27$ & $22 \pm 0.34$ & $28 \pm 0.59$ & $25 \pm 0.46$ & $17 \pm 0.37$ & $41 \pm 1.4$ & -0.87 & II & $\mathrm{Br}, \mathrm{Pa} \beta$, Sp.Obj. "M" \\
\hline J053354.13+371900.3 & $\ldots$ & $\ldots$ & . & $1.3 \pm 0.038$ & $5.6 \pm 0.12$ & $13 \pm 0.29$ & $58 \pm 1.9$ & 0.77 & I & $\mathrm{C}$ \\
\hline J053354.70+372202.5 & $\ldots$ & $\ldots$ & . & $0.61 \pm 0.018$ & $2.3 \pm 0.058$ & $5.8 \pm 0.22$ & $44 \pm 1.6$ & 1.00 & I & \\
\hline J053355.75+372317.8 & $0.056 \mathrm{u}$ & $0.25 \mathrm{u}$ & $1.3 \pm 0.1$ & $4.2 \pm 0.1$ & $11 \pm 0.24$ & $6.2 \pm 0.25$ & $23 \pm 1.3$ & -0.08 & Flat & $\mathrm{C}$ \\
\hline J053356.07+372302.6 & $0.18 \mathrm{u}$ & $0.88 \pm 0.082$ & $2.6 \pm 0.12$ & $3.6 \pm 0.093$ & $6.2 \pm 0.17$ & $7 \pm 0.3$ & $21 \pm 2.1$ & -0.21 & Flat & C, Sp.Obj. "E" \\
\hline J053357.35+372318.1 & $0.36 \mathrm{u}$ & $1.9 \pm 0.082$ & $5.6 \pm 0.14$ & $11 \pm 0.22$ & $18 \pm 0.33$ & $23 \pm 0.45$ & $72 \pm 1.8$ & -0.05 & Flat & $\mathrm{C}, \mathrm{Br}, \mathrm{Pa} \beta$, Sp.Obj. "D" \\
\hline J053358.01+372347.0 & $\ldots$ & $\ldots$ & $\ldots$ & $0.89 \pm 0.023$ & $3.3 \pm 0.074$ & $2.6 \pm 0.19$ & $16 \pm 1.4$ & 0.18 & Flat & $\mathrm{C}$ \\
\hline J053359.08+372410.3 & $0.11 \mathrm{u}$ & $0.098 \mathrm{u}$ & $1.2 \pm 0.1$ & $3.2 \pm 0.067$ & $6.1 \pm 0.13$ & $3.5 \pm 0.18$ & $21 \pm 1.3$ & -0.08 & Flat & $\mathrm{C}$ \\
\hline J053400.05+372426.6 & $0.29 \mathrm{u}$ & $3 \pm 0.11$ & $11 \pm 0.24$ & $11 \pm 0.22$ & $15 \pm 0.28$ & $11 \pm 0.28$ & $160 \pm 2.9$ & -0.10 & Flat & C, Sp.Obj. "A" \\
\hline J053400.05+372442.6 & $\ldots$ & $\ldots$ & $\ldots$ & $1.7 \pm 0.04$ & $6.1 \pm 0.12$ & $3.8 \pm 0.19$ & $21 \pm 1.2$ & -0.04 & Flat & $\mathrm{C}$ \\
\hline J053401.29+372357.9 & $\ldots$ & $\ldots$ & $\ldots$ & $0.41 \pm 0.046$ & $0.54 \pm 0.058$ & $5.5 \pm 0.25$ & $17 \pm 1.7$ & 1.09 & I & C, Sp.Obj. "C" \\
\hline J053401.79+372500.6 & $\ldots$ & $\ldots$ & $\ldots$ & $0.67 \pm 0.02$ & $5.3 \pm 0.11$ & $2.3 \pm 0.17$ & $87 \pm 2.2$ & 0.91 & I & $\mathrm{C}$ \\
\hline J053410.97+372725.0 & $1.5 \pm 0.063$ & $3.3 \pm 0.093$ & $4.3 \pm 0.12$ & $4.6 \pm 0.11$ & $4.5 \pm 0.095$ & $3.4 \pm 0.19$ & $17 \pm 1$ & -0.57 & II & $\mathrm{N}, \mathrm{C}$ \\
\hline J053418.99+372038.7 & $200 \pm 3.3$ & $420 \pm 6.3$ & $440 \pm 6$ & $280 \pm 6.1$ & $150 \pm 2.9$ & $31 \pm 0.53$ & $15 \pm 1$ & -2.52 & III & \\
\hline J053425.88+372631.1 & $170 \pm 2.8$ & $290 \pm 4.3$ & $260 \pm 3.7$ & $150 \pm 3.6$ & $80 \pm 1.5$ & $16 \pm 0.4$ & $11 \pm 1$ & -2.43 & III & \\
\hline J053429.31+372651.4 & $440 \pm 7.7$ & $480 \pm 11$ & $400 \pm 5.5$ & $270 \pm 6.4$ & $190 \pm 3.3$ & $51 \pm 0.75$ & $27 \pm 1.2$ & -2.20 & III & \\
\hline J053430.01+372120.8 & $0.8 \mathrm{u}$ & $1 \mathrm{u}$ & $0.77 \pm 0.1$ & $1.1 \pm 0.036$ & $4 \pm 0.093$ & $12 \pm 0.28$ & $64 \pm 2.3$ & 0.86 & I & $\mathrm{C}$ \\
\hline J053430.25+372141.7 & $0.66 \pm 0.058$ & $1.2 \pm 0.079$ & $1.7 \pm 0.1$ & $1.9 \pm 0.044$ & $2.4 \pm 0.059$ & $3.8 \pm 0.2$ & $10 \pm 1$ & -0.27 & Flat & $\mathrm{C}$ \\
\hline J053432.15+372643.8 & $770 \pm 13$ & $930 \pm 17$ & $700 \pm 11$ & $360 \pm 9.7$ & $180 \pm 3.4$ & $38 \pm 0.62$ & $16 \pm 1$ & -2.65 & III & \\
\hline J053440.62+371050.5 & .. & .. & & $1.1 \pm 0.029$ & $8.9 \pm 0.17$ & $16 \pm 0.32$ & $210 \pm 3.8$ & 1.31 & I & \\
\hline J053447.31+373555.5 & $0.98 \pm 0.05$ & $0.9 \pm 0.063$ & $0.69 \pm 0.079$ & $0.72 \pm 0.022$ & $0.69 \pm 0.029$ & $17 \pm 0.4$ & $28 \pm 1.7$ & 0.88 & I & $\mathrm{C}$ \\
\hline
\end{tabular}

Notes.

a The WISE designation has the format Jhhmmss.ss \pm ddmmss.s.

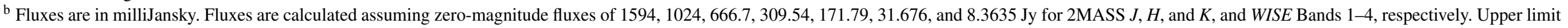
measurements are denoted with a " $u$ " following the flux.

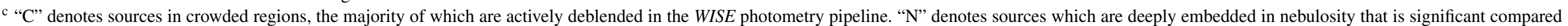

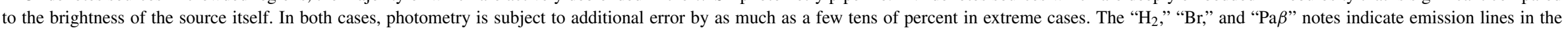

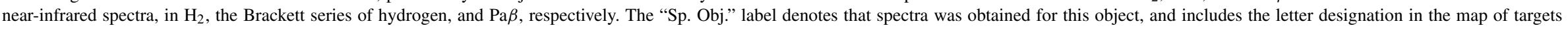
(Figure 6) 


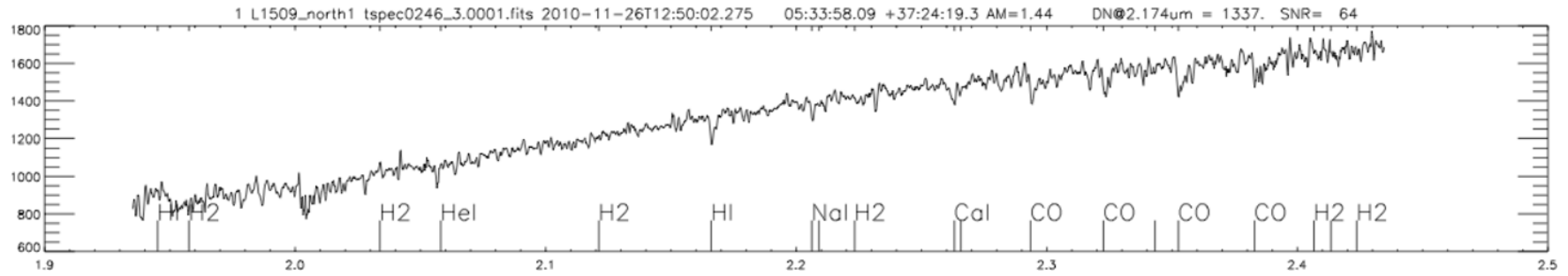

Figure 7. Spectra of WISE J053400.05+372426.6, denoted object "A" in Figure 6. In this figure, and the 18 that follow, the portion of the spectrum for which we have obtained S/N > 10 is shown. Locations of lines for atomic and molecular species common to YSOs are shown for reference, and do not necessarily indicate that a line was detected at that location.
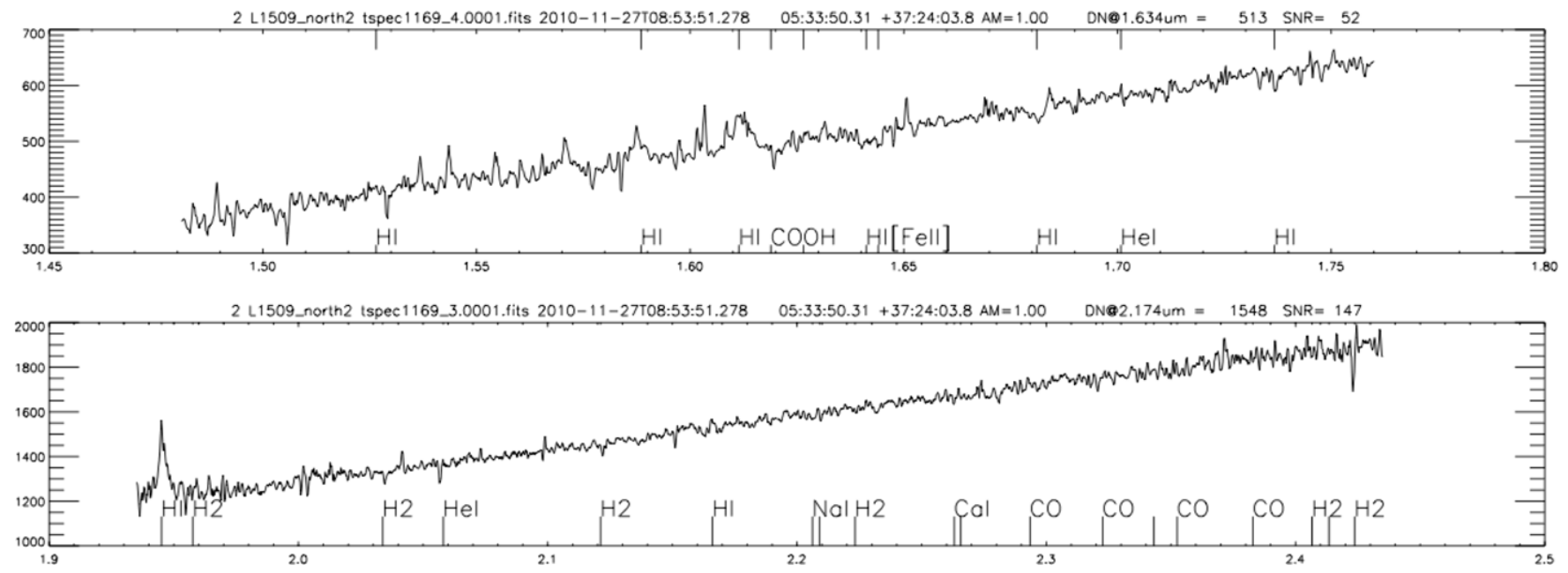

Figure 8. Spectra of WISE J053401.29+372357.9, denoted object "B" in Figure 6.
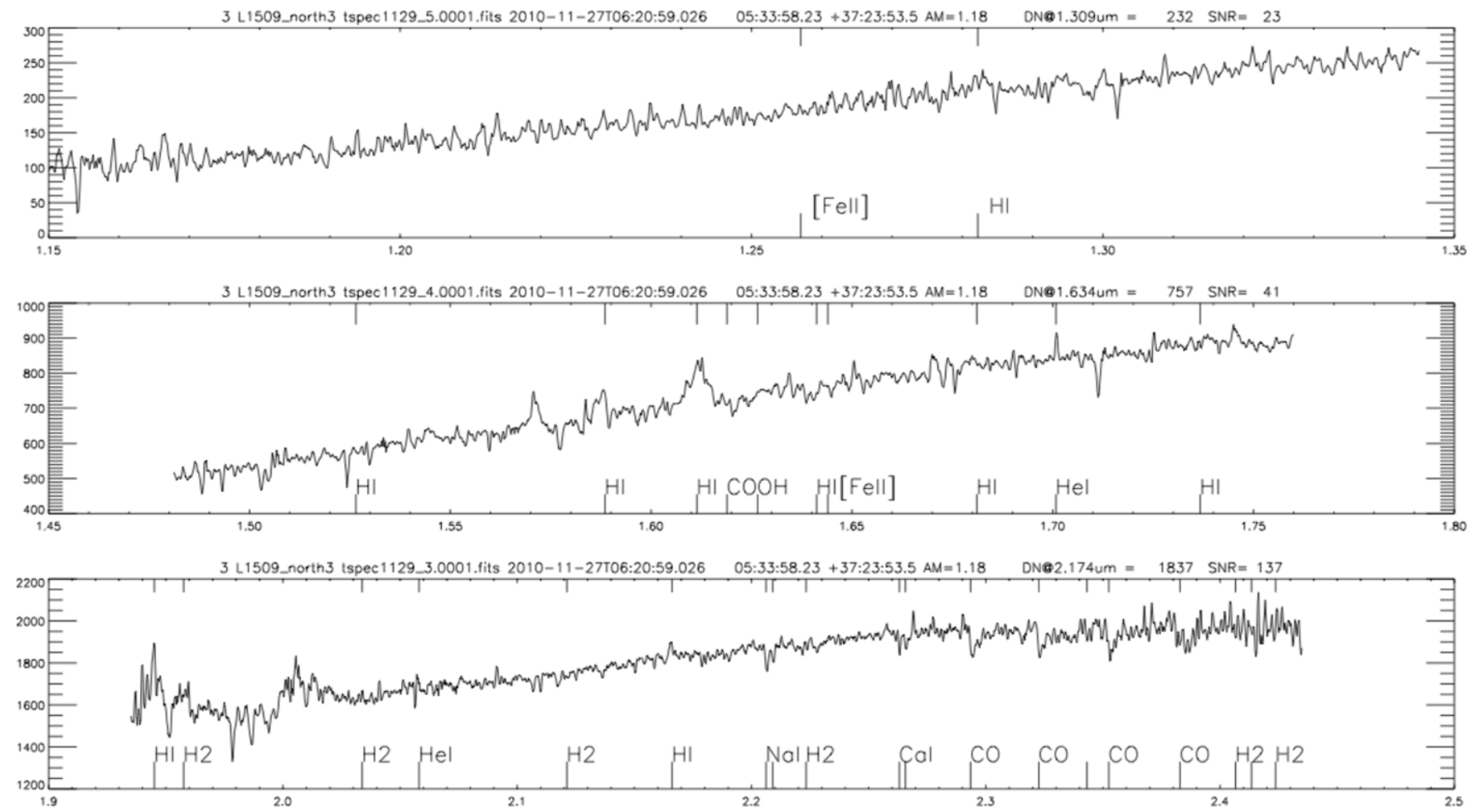

Figure 9. Spectra of WISE J053357.35+372318.1, denoted object "C" in Figure 6.

\section{DISCUSSION}

\subsection{The Distance to $L 1509$}

The distance to L1509 is uncertain. Previous literature on L1509 is sparse, though there have been some large-area surveys of molecular clouds that cover the region. One such study quotes a distance of $2 \mathrm{kpc}$ and quotes a value of $v_{\mathrm{LSR}}=21 \mathrm{~km} \mathrm{~s}^{-1}$ (Kawamura et al. 1998), which would place it in the Perseus arm of the galaxy. This would make the region an order of magnitude more distant than the Taurus-Auriga star forming 

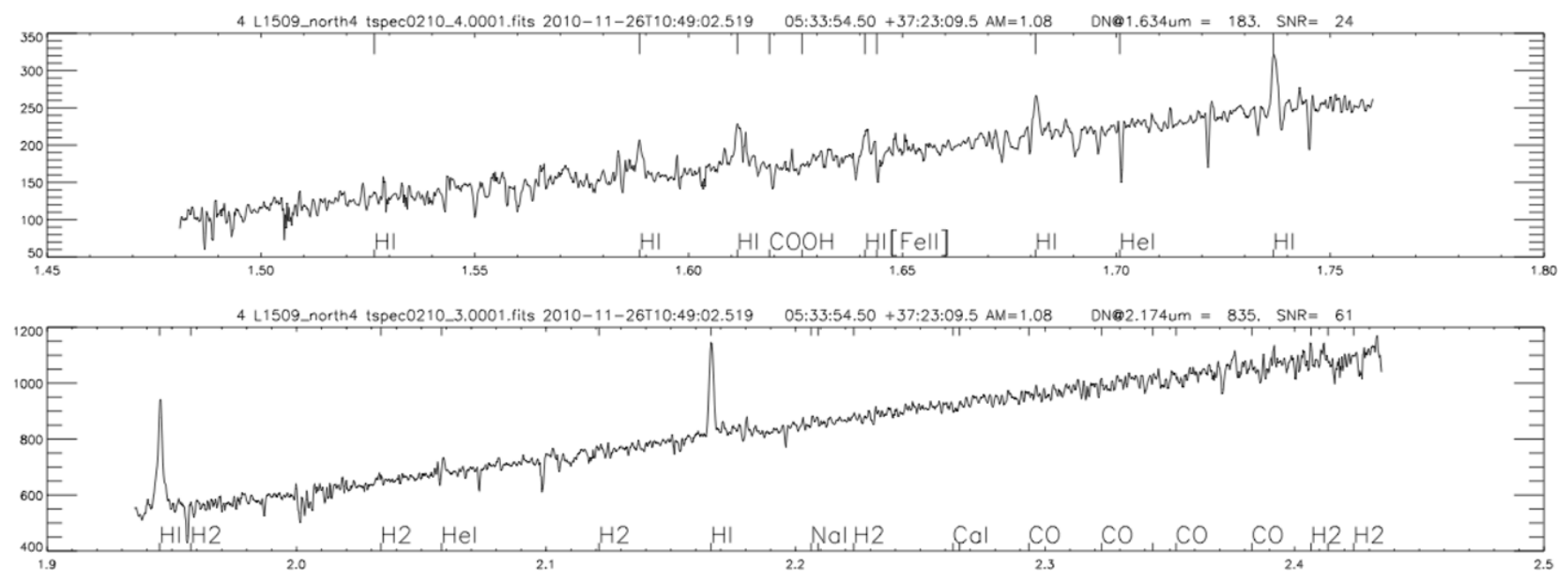

Figure 10. Spectra of WISE J053355.75+372317.8, denoted object "D" in Figure 6.

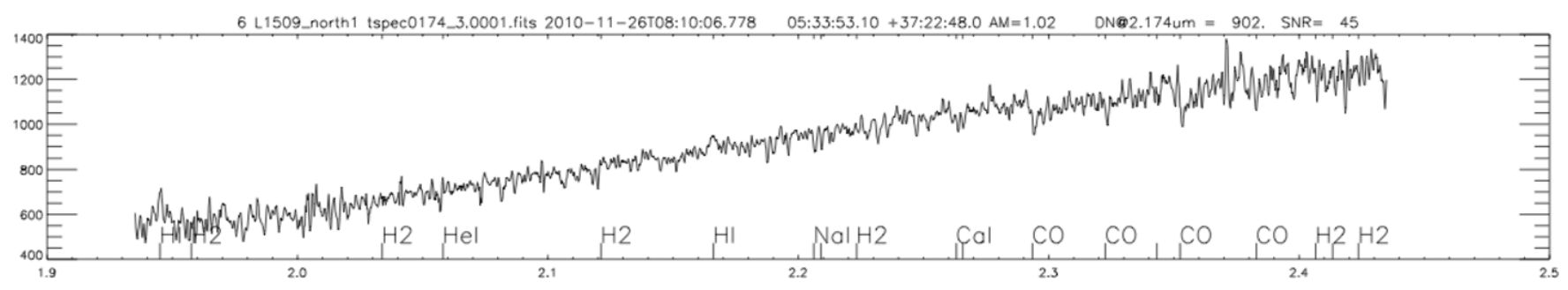

Figure 11. Spectra of WISE J053356.07+372302.6, denoted object "E" in Figure 6.

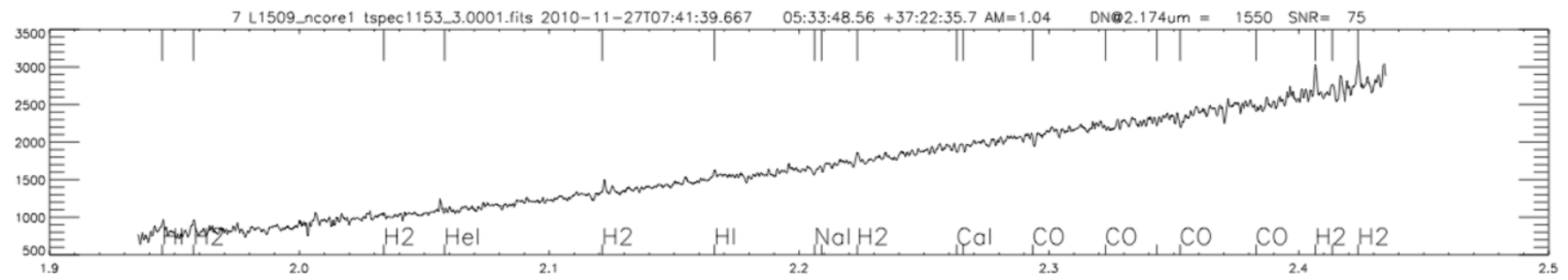

Figure 12. Spectra of WISE J053351.39+372246.1, denoted object "F" in Figure 6.
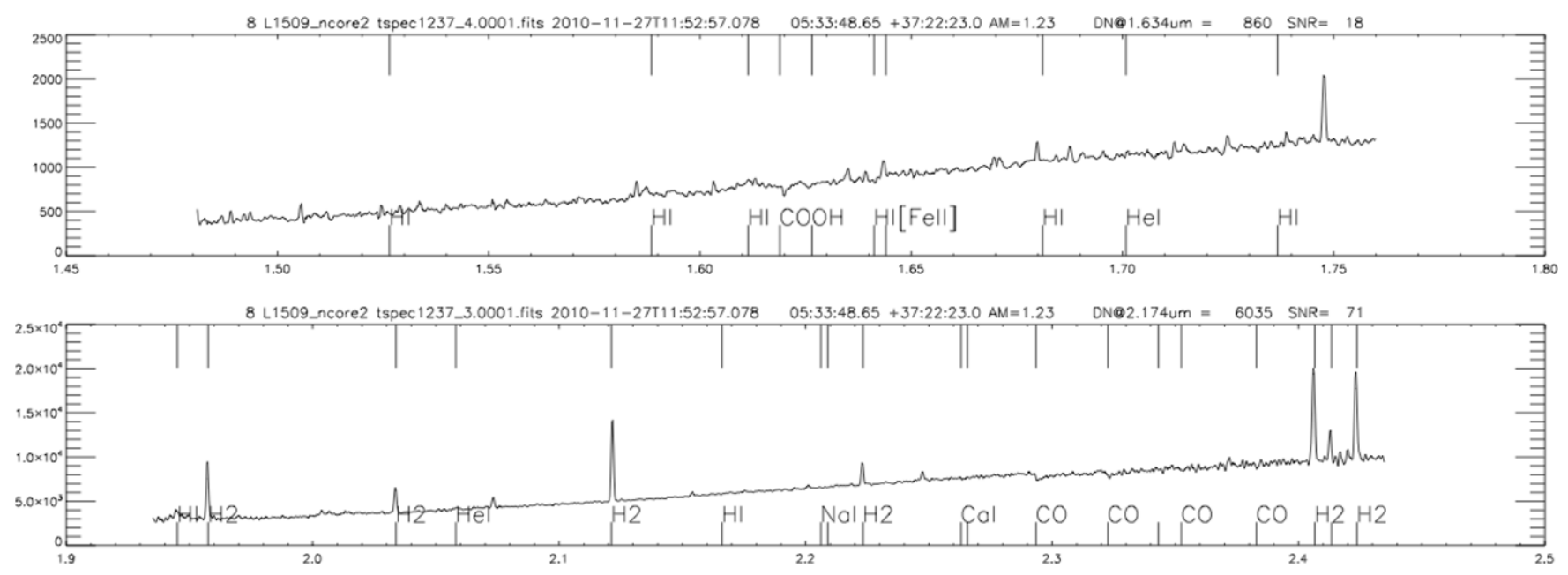

Figure 13. Spectra of WISE J053351.40+372226.6, denoted object "G" in Figure 6. 

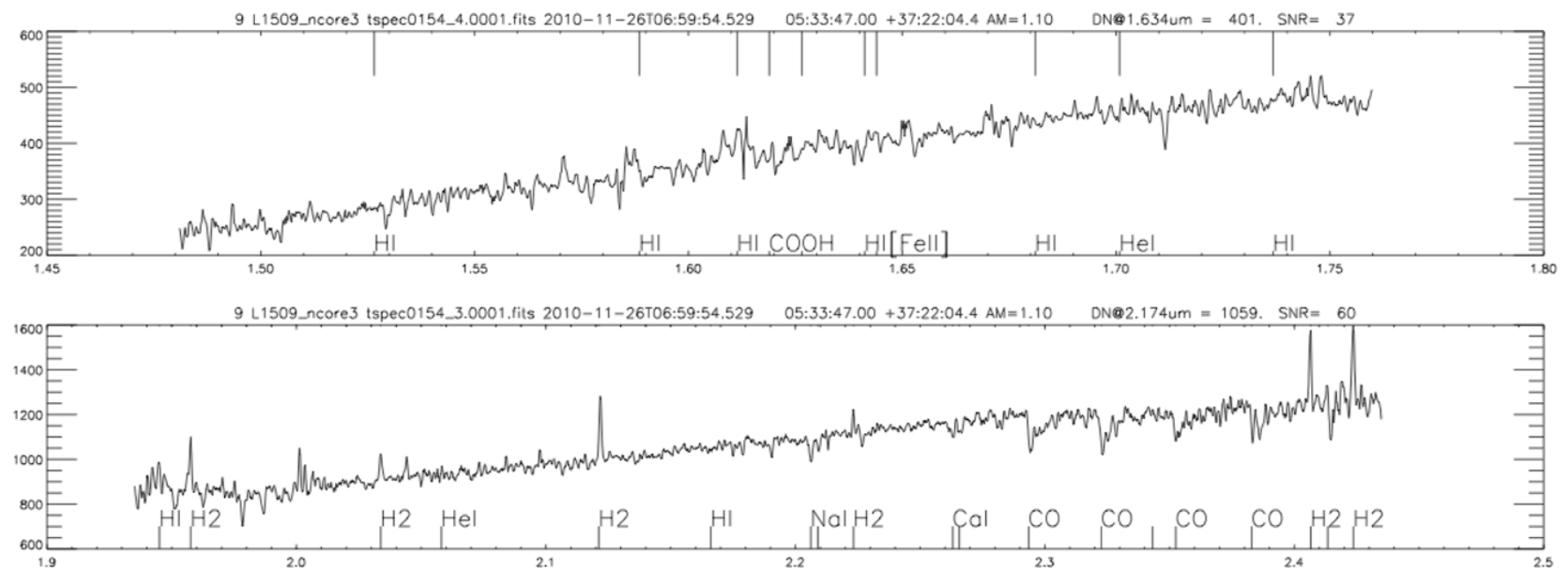

Figure 14. Spectra of WISE J053350.04+372225.4, denoted object "H" in Figure 6.

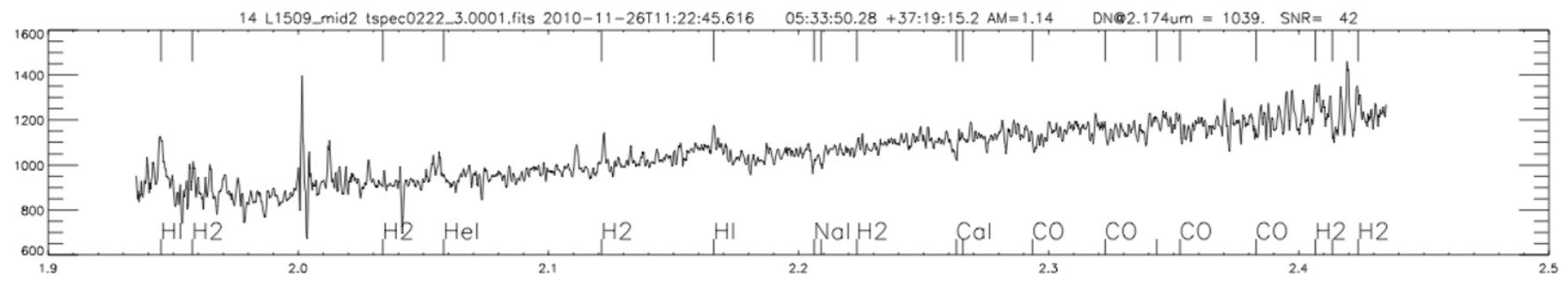

Figure 15. Spectra of WISE J053352.99+371923.7, denoted object "I" in Figure 6.
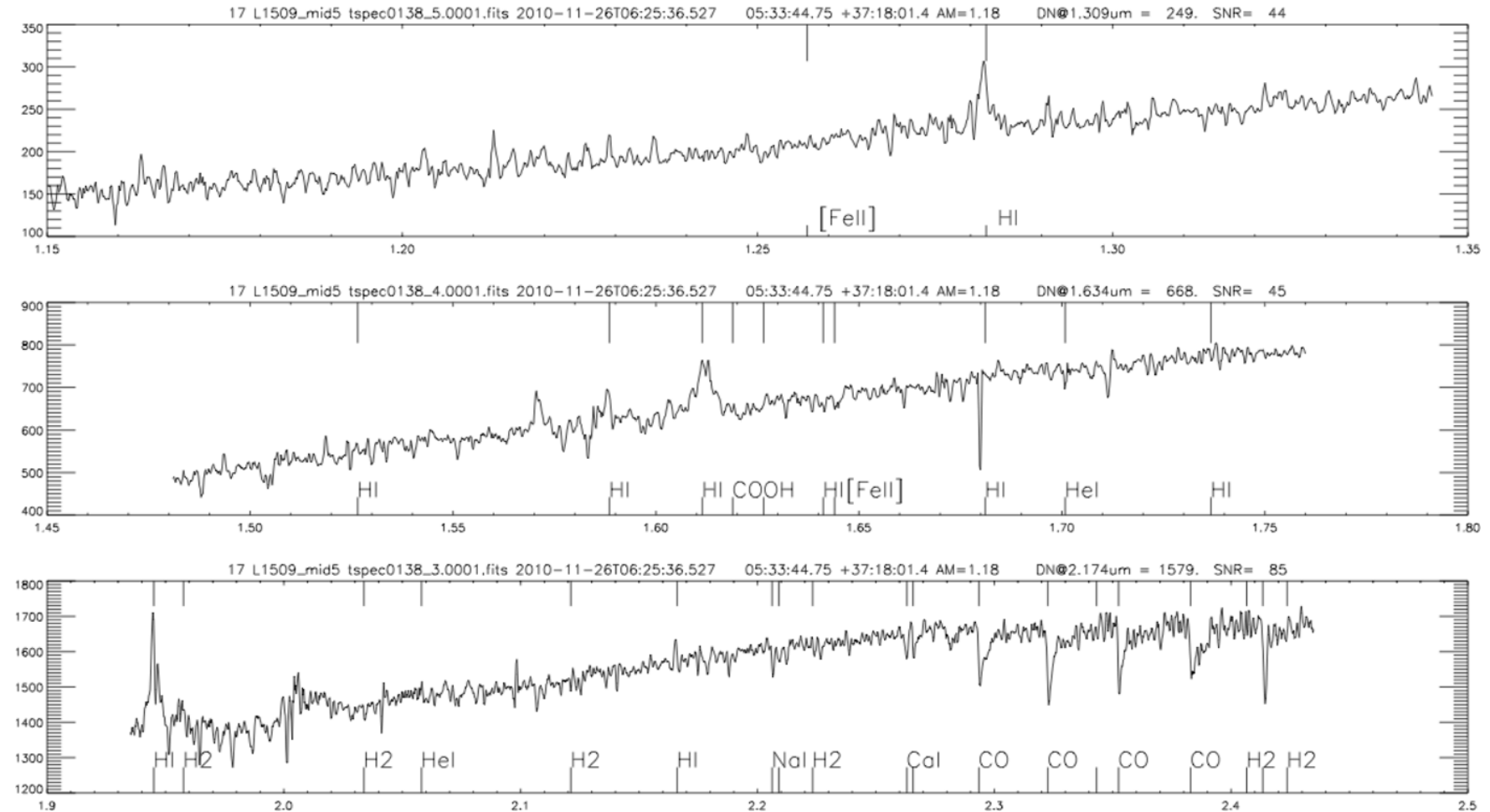

Figure 16. Spectra of WISE J053348.09+371820.1, denoted object "J" in Figure 6.

region, at $140 \mathrm{pc}$ (Kenyon et al. 2008). In order to assess which of these distances is more reasonable for the L1509 cluster, we make an order of magnitude estimate for the distance to the cluster by using our spectroscopic data and WISE photometry.

The K-type stars presented in Section 3 in the have average apparent magnitudes in $W 1$ of $11.2 \pm 0.8$, and $W 2$ of $9.9 \pm 0.7$.
It should be noted that the errors in these values represent the large intrinsic dispersion in YSO brightnesses, due to disk geometry, viewing angle, etc., and not photometric errors. In the Taurus-Auriga region, there are 32 confirmed member YSOs with spectral types from K1 through K8 and SED classes of I, flat spectrum, or II (Rebull et al. 2010). The Taurus-Auriga stars 


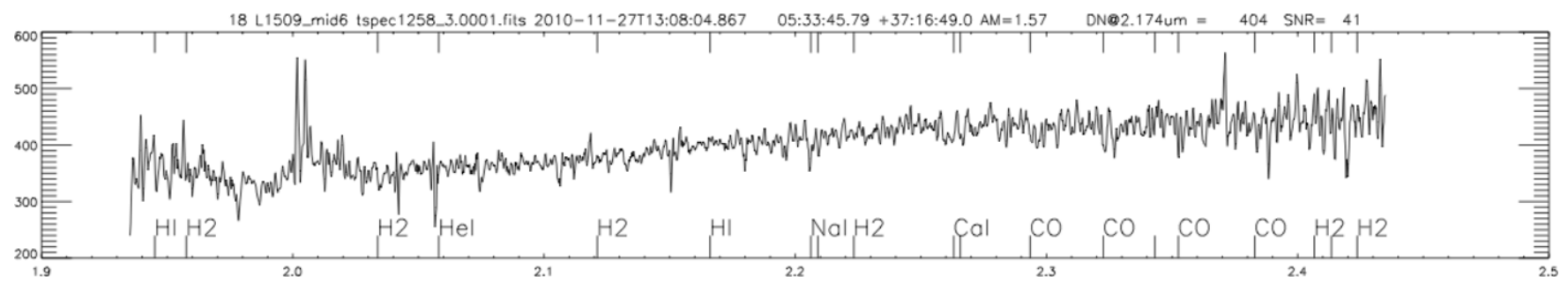

Figure 17. Spectra of WISE J053347.35+371652.9, denoted object "K" in Figure 6.

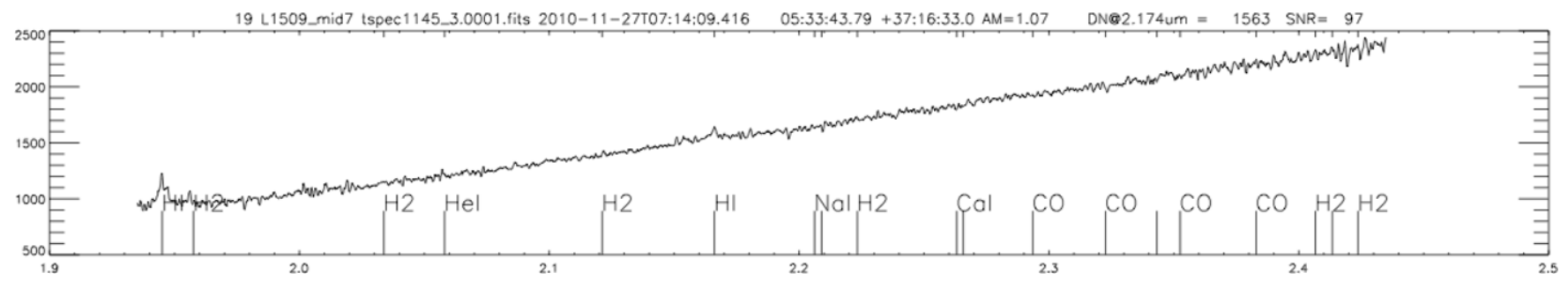

Figure 18. Spectra of WISE J053346.60+371644.4, denoted object "L" in Figure 6.
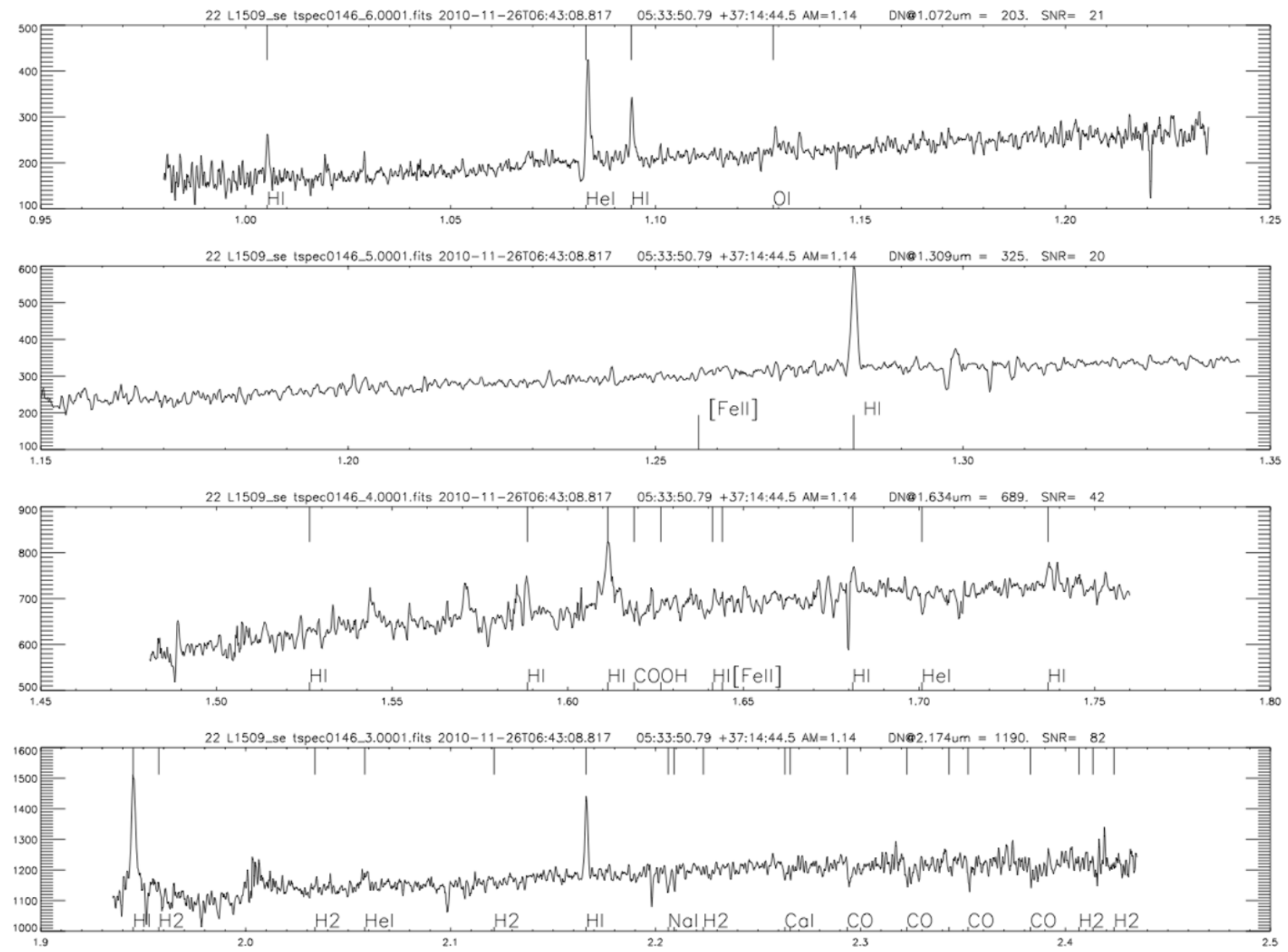

Figure 19. Spectra of WISE J053354.02+371503.1, denoted object “M" in Figure 6.

have average IRAC magnitudes of $7.7 \pm 1.5$ at [3.6] and $7.2 \pm$ 1.5 at $[4.5] .^{8}$

8 The saturation limits for IRAC are 6.6 at $3.5 \mu \mathrm{m}$ and 6.1 at $4.5 \mu \mathrm{m}$. About a third of the objects are brighter than the saturation limit at one of these wavelengths. For the calculation of the average, we adopt the saturation limits of 6.6 and $6.1 \mathrm{mag}$ for [3.6] and [4.5], respectively, for these sources. This will
If we take $W 1$ to be equivalent to the IRAC $3.6 \mu \mathrm{m}$ band and $W 2$ to be equivalent to IRAC $4.5 \mu \mathrm{m}$, we can use the magnitude differences to estimate the distance to the cluster

result in an underestimate in average brightness of the Taurus stars, but the result is still useful for an order of magnitude estimate. 

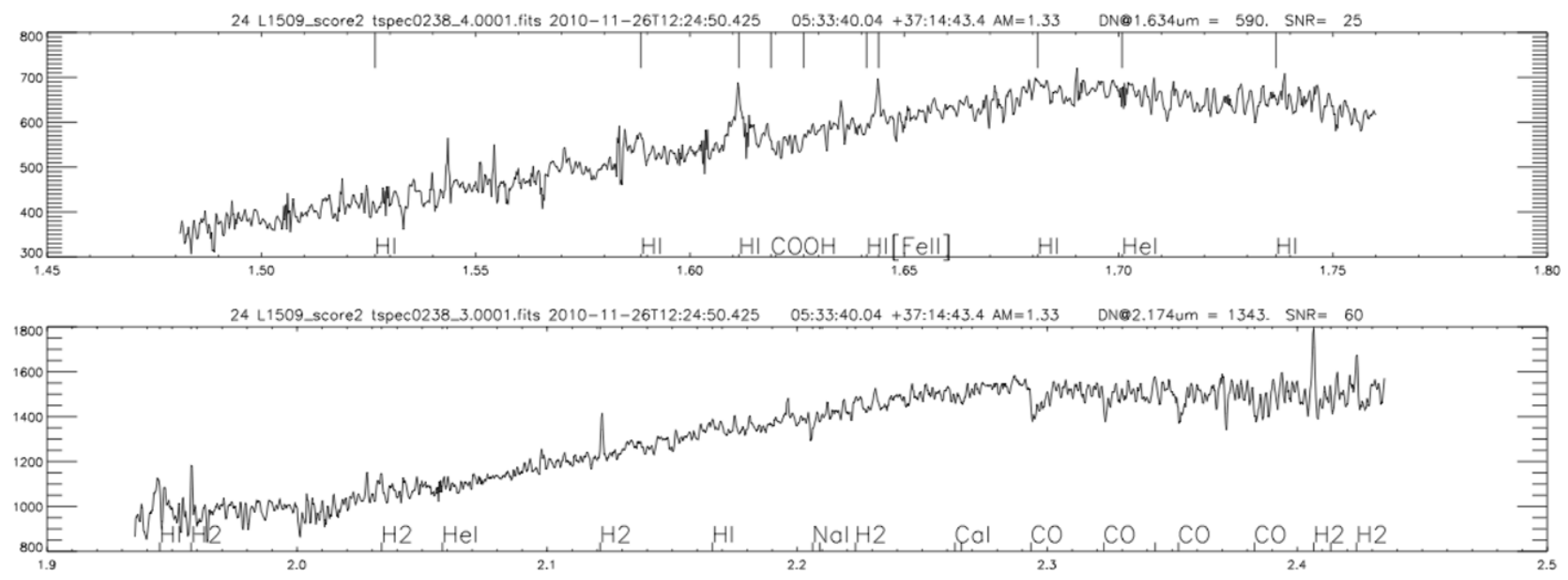

Figure 20. Spectra of WISE J053342.32+371450.2, denoted object "O" in Figure 6.
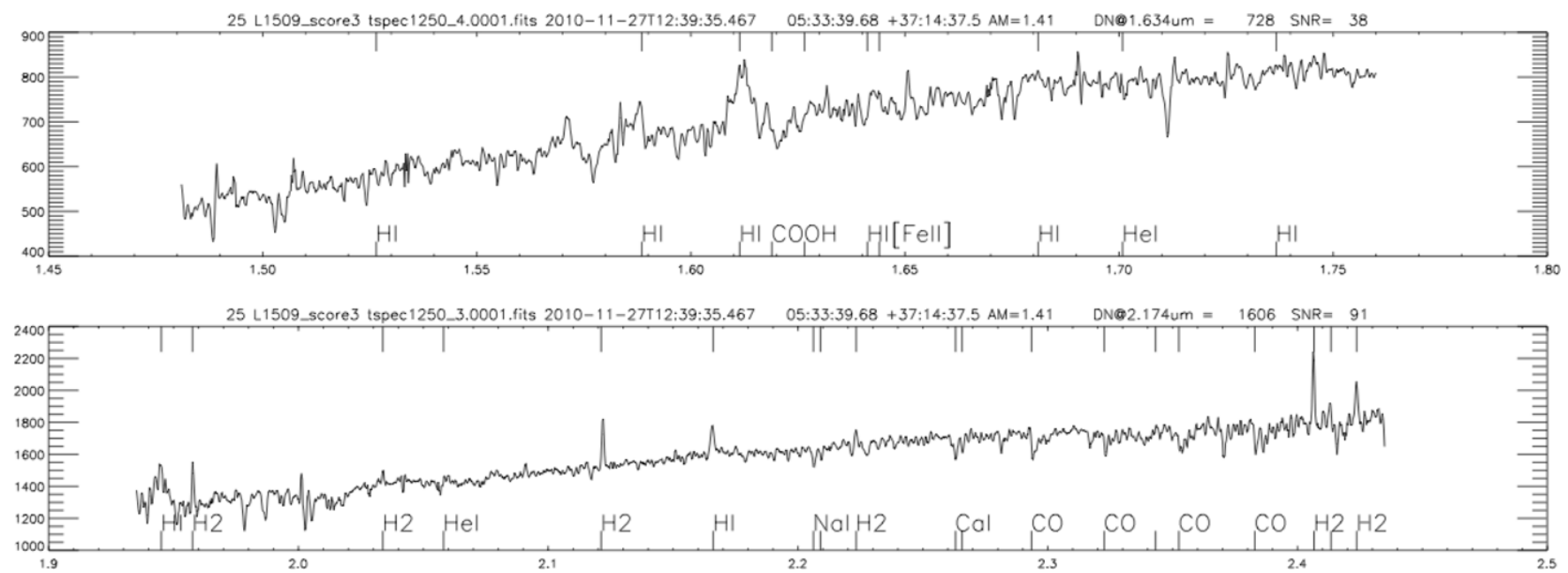

Figure 21. Spectra of WISE J053342.05+371439.2, denoted object "P" in Figure 6.

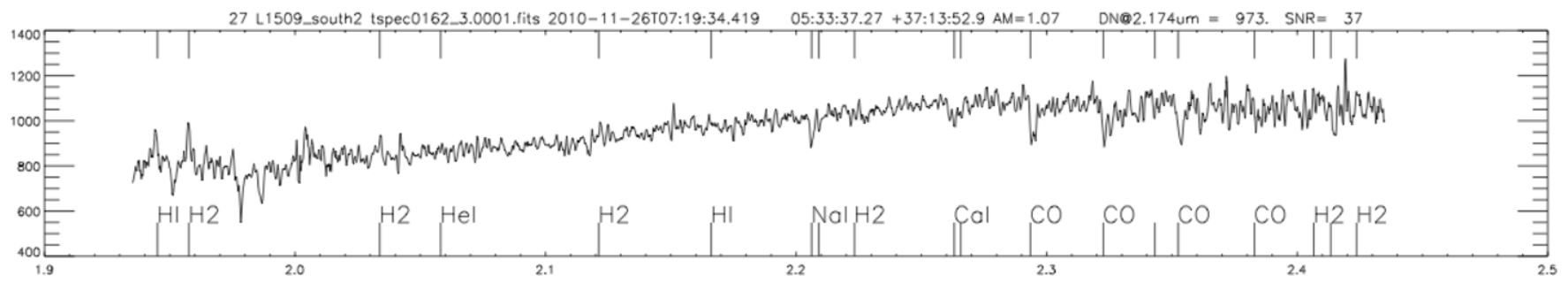

Figure 22. Spectra of WISE J053340.14+371408.6, denoted object "Q" in Figure 6.

Table 2

K Dwarfs in L1509

\begin{tabular}{lccc}
\hline \hline Source ID & $\begin{array}{c}\text { EW(Na I) + EW(Ca I) } \\
(\AA)\end{array}$ & $\begin{array}{c}\text { EW(CO) } \\
(\AA)\end{array}$ & Figure 6 Label \\
\hline J053400.05+372426.6 & 5.4 & 3.0 & A \\
J053401.29+372357.9 & 4.3 & 2.3 & $\mathrm{C}$ \\
J053356.07+372302.6 & 5.7 & 4.3 & $\mathrm{E}$ \\
J053350.04+372225.4 & 6.6 & 3.1 & $\mathrm{H}$ \\
J053340.14+371408.6 & 9.3 & 5.4 & $\mathrm{Q}$ \\
\hline
\end{tabular}

$(W 1-[3.6]=3.5 \pm 1.7$ and $W 2-[4.5]=2.7 \pm 1.7)$. Using $W 1-[3.6]=3.5$ results in a distance of about $700 \mathrm{pc}$. Using the $W 2-[4.5]=2.7$ difference results in a distance of $485 \mathrm{pc}$.
Accounting for the errors on the magnitudes, the cluster can lie anywhere between $350 \mathrm{pc}$ and $1500 \mathrm{pc}$. If the YSOs from the Taurus region are placed at a distance of $2 \mathrm{kpc}$, they would have apparent magnitudes fainter by $5.8 \mathrm{mag}$, which is somewhat outside our estimate. However, though our estimate is the L1509 cluster is likely closer than the previously estimated distance of $2 \mathrm{kpc}$, we cannot rule out a $2 \mathrm{kpc}$ distance, given the large intrinsic dispersion in YSO luminosities, even those with similar spectral classes.

An alternative possibility that cannot be ruled out is that the L1509 cloud lies at a distance of $2 \mathrm{kpc}$, but that the YSO population is comprised of early-type stars intrinsically more massive and luminous than the Taurus YSOs. The spectral types obtained here are relatively uncertain and some of the 

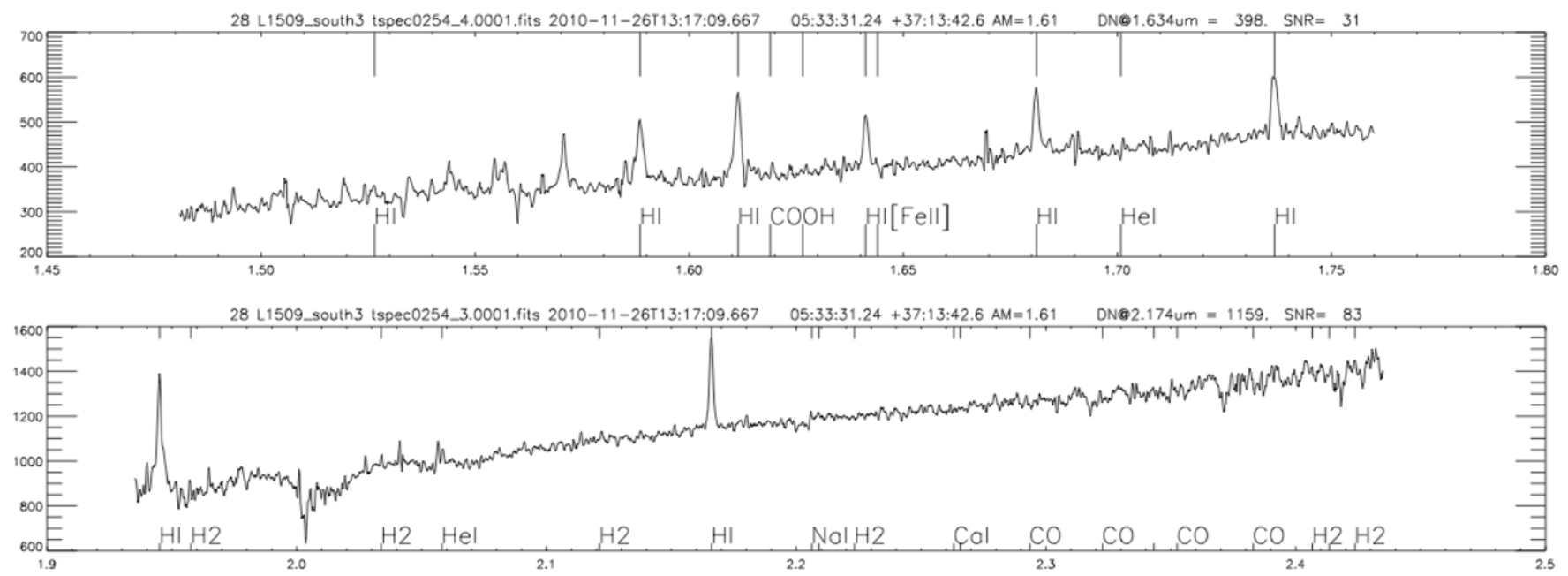

Figure 23. Spectra of WISE J053332.75+371349.9, denoted object "R" in Figure 6.
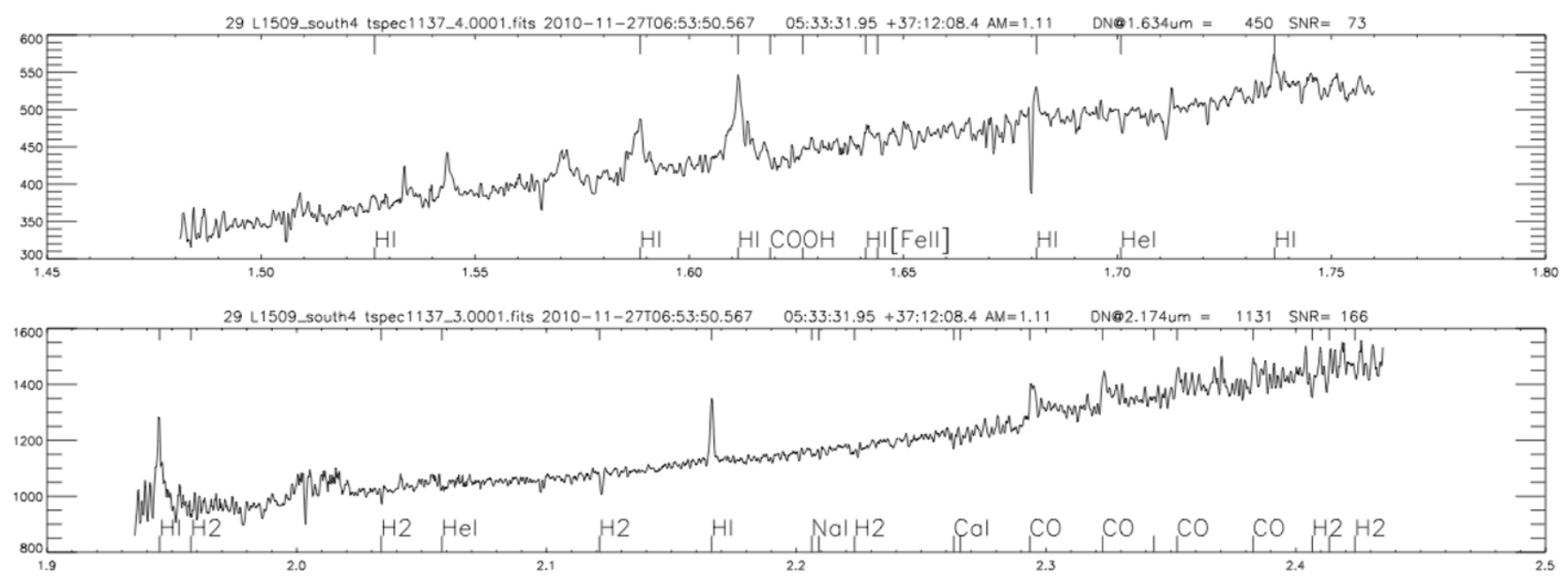

Figure 24. Spectra of WISE J053334.80+371219.6, denoted object "S" in Figure 6.

late-type objects could be evolved giants, although their spatial coincidence with a dark cloud and emission lines in their spectra make this unlikely. It should be noted that we do not account for extinction in the L1509 sample, which would tend to make these sources closer, although extinction effects are minimized at the wavelengths of WISE.

\subsection{Comparison to Other Star Forming Regions}

The distribution of YSOs in the L1509 cluster displays some similarities to other well-studied star forming regions. Qualitatively, the reddest objects are clustered most tightly along the filamentary aggregate, while a larger proportion of Class II objects are found outside the most densely populated regions. To illustrate this quantitatively we calculate the median separation between closest neighbors of a given YSO class. We find that the Class II objects have the greatest median closest-neighbor separation $\left(78^{\prime \prime}\right)$, followed by Class I and flat spectrum objects (45" and $26^{\prime \prime}$, respectively). The higher proportion of Class I and flat spectrum objects in the densest clusters is similar to the recent findings for several clusters at close and intermediate distances, including the Western Circinus YSO cluster (700 pc; Liu et al. 2011), Serpens (260 pc; Harvey et al. 2007), and the "Gulf of Mexico" cluster in the
North American Nebula, (600 pc; Rebull et al. 2011a). Like Harvey et al. (2007) we believe this suggests that there may be a number of older, more evolved objects in addition to the youngest YSOs.

If we assume the closest possible distance for L1509, $350 \mathrm{pc}$, we can compare the cluster to the Lynds clouds in the Perseus star forming complex. The Perseus complex lies at a distance of about $300 \mathrm{pc}$ and contains numerous dark clouds and associated clusters of YSOs, spanning the range of pre-main sequence evolution (Bally et al. 2008). The regions IC 348, NGC 1333 and several Lynds dark clouds (1451, 1455, 1448). NGC 1333 and the Lynds clouds in Perseus all display signs of relative youth, including a large fraction of Class I objects, and protostellar outflows. The large fraction of Class I objects in L1509 (about $34 \%$ of mid-infrared excess objects characterized in this study) indicates its youth and star formation activity. Our spectroscopic data also confirms the youth of the region, with several sources showing strong emission lines in the Brackett series (indicative of active accretion), $\mathrm{Pa} \beta$, and $\mathrm{H}_{2}$ emission, the latter indicative of shock-excited emission (Beck et al. 2008). There is also one source with CO $(2.293-2.414 \mu \mathrm{m})$ in emission, indicative of veiling (Connelley \& Greene 2010). Given these indicators of youth, L1509 is similar to the smaller Perseus aggregates (outside of IC 348 and NGC 1333), in which nearly half of the 
YSOs are Class I or flat spectrum objects. In contrast, the older IC 348 is dominated by the more evolved Class II objects, with only $14 \%$ of the YSOs classified as Class I or flat spectrum sources (Bally et al. 2008).

A survey of molecular clouds by Kawamura et al. (1998) shows a $13 \mathrm{CO}$ cloud (named cloud 10) by the study centered about $40^{\prime}$ north of L1509, and a $v_{\mathrm{LSR}}=-21 \mathrm{~km} \mathrm{~s}^{-1}$, placing it in the Persues arm of the galaxy, at a distance of $2 \mathrm{kpc}$. It is uncertain whether L1509 is physically associated with this cloud, as they do not appear to overlap given their positions and sizes. However, we cannot definitively rule out a $2 \mathrm{kpc}$ distance for the L1509 cluster. Assuming a larger distance for the L1509 forces the stellar population of the cluster to be comprised of earlier-type objects than other nearby low-mass star forming regions, in order for the apparent magnitudes to be consistent. Well-studied star forming regions between 1.5 and $2 \mathrm{kpc}$ include Cygnus X (1700 pc; Reipurth \& Schneider 2008) and the Rosette complex, including NGC 2244 (1600-1700 pc; Román-Zúñiga $\&$ Lada 2008). Regions at these distances are dominated by high-mass protostars of spectral type $\mathrm{O}$ and B. Such massive protostars are not evident in the observations.

\section{CONCLUSIONS}

WISE observations have uncovered a population of YSOs associated with the L1509 dark cloud. Analysis of photometry from the near-infrared to the mid-infrared shows 55 Class I, II, and flat spectrum objects with circumstellar disks. We find that the reddest objects comprise the densest YSO aggregates in the cluster, while Class II objects are more widely distributed. Using $K$-band spectroscopy, we find that the likely distance to this cluster is between 350 and $1500 \mathrm{pc}$. Based on the large relative number of Class I objects (over one-third of YSO candidates), we conclude that the L1509 region is young and actively forming stars, comparable to the Lynds dark clouds in the Perseus complex. We also conclude that L1509 is a physically distinct region, not physically associated with the Taurus-Auriga complex, and likely at least a factor of two more distant.

This publication makes use of data products from the Widefield Infrared Survey Explorer, a joint project of the University of California, Los Angeles, and the Jet Propulsion Laboratory/ California Institute of Technology, funded by the National
Aeronautics and Space Administration. This publication makes use of data products from the Two Micron All Sky Survey, which is a joint project of the University of Massachusetts and the Infrared Processing and Analysis Center/California Institute of Technology, funded by the National Aeronautics and Space Administration and the National Science Foundation. W.M.L. acknowledges support from WISE and the WISE Science Data Center. This research made use of the SIMBAD database.

\section{REFERENCES}

Angione, J. R. 2012, M.S. thesis, California State Univ., Los Angeles

Bally, J., Walawender, J., Johnstone, D., Kirk, H., \& Goodman, A. 2008, in Handbook of Star Forming Regions, Volume I, ed. B. Reipurth (San Francisco, CA: ASP), 308

Barnard, E. E. 1919, ApJ, 49, 1

Beck, T. L., McGregor, P. J., Takami, M., \& Pyo, T.-S. 2008, ApJ, 676, 472 Carey, S. J., Noriega-Crespo, A., Mizuno, D. R., et al. 2009, PASP, 121, 76 Churchwell, E., Babler, B. L., Meade, M. R., et al. 2009, PASP, 121, 213

Connelley, M. S., \& Greene, T. P. 2010, AJ, 140, 1214

Cutri, R. M., Wright, E. L., Conrow, T., et al. 2012, Explanatory Supplement to the WISE All-Sky Data Release Products, 1

Dobashi, K., Uehara, H., Kandori, R., et al. 2005, PASJ, 57, 1

Dutra, C. M., \& Bica, E. 2002, A\&A, 383, 631

Greene, T. P., \& Lada, C. J. 1996, AJ, 112, 2184

Harvey, P., Merín, B., Huard, T. L., et al. 2007, ApJ, 663, 1149

Kawamura, A., Onishi, T., Yonekura, Y., et al. 1998, ApJS, 117, 387

Kenyon, S. J., Gómez, M., \& Whitney, B. A. 2008, in Handbook of Star Forming Regions, Volume I, ed. B. Reipurth (San Francisco, CA: ASP), 405

Kiss, C., Moór, A., \& Tóth, L. V. 2004, A\&A, 418, 131

Koenig, X. P., Leisawitz, D. T., Benford, D. J., et al. 2012, ApJ, 744, 130

Lada, C. J., Alves, J. F., \& Lombardi, M. 2007, in Protostars and Planets V, ed. B. Reipurth, D. Jewitt, \& K. Keil (Tucson, AZ: Univ. Arizona Press), 3

Liu, W. M., Padgett, D. L., Leisawitz, D., Fajardo-Acosta, S., \& Koenig, X. P. 2011, ApJL, 733, L2

Lynds, B. T. 1962, ApJS, 7, 1

Rebull, L. M., Guieu, S., Stauffer, J. R., et al. 2011a, ApJS, 193, 25

Rebull, L. M., Koenig, X. P., Padgett, D. L., et al. 2011b, ApJS, 196, 4

Rebull, L. M., Padgett, D. L., McCabe, C.-E., et al. 2010, ApJS, 186, 259

Reipurth, B., \& Schneider, N. 2008, in Handbook of Star Forming Regions, Volume I, ed. B. Reipurth (San Francisco, CA: ASP), 36

Román-Zúñiga, C. G., \& Lada, E. A. 2008, in Handbook of Star Forming Regions, Volume I, ed. B. Reipurth (San Francisco, CA: ASP), 928

Skrutskie, M. F., Cutri, R. M., Stiening, R., et al. 2006, AJ, 131, 1163

White, R. J., Greene, T. P., Doppmann, G. W., Covey, K. R., \& Hillenbrand, L. A. 2007, in Protostars and Planets V, ed. B. Reipurth, D. Jewitt, \& K. Keil (Tucson, AZ: Univ. Arizona Press), 117

Wilking, B. A., Bontemps, S., Schuler, R. E., Greene, T. P., \& André, P. 2001, ApJ, 551, 357

Wright, E. L., Eisenhardt, P. R. M., Mainzer, A. K., et al. 2010, AJ, 140, 1868 